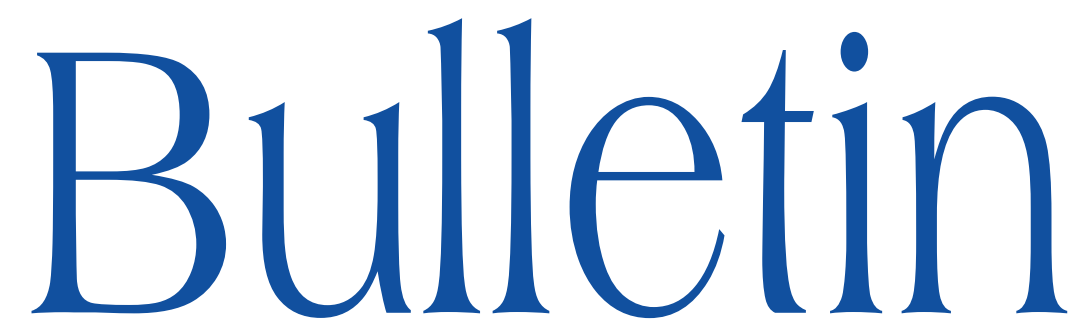

de la SOCIÉTÉ MATHÉMATIQUE DE FRANCE

\title{
INTEGRAL REPRESENTATIONS FOR SOLUTIONS OF EXPONENTIAL GAUSS-MANIN SYSTEMS
}

Marco Hien \& Céline Roucairol

Tome 136

Fascicule 4

2008

SOCIÉTÉ MATHÉMATIQUE DE FRANCE

Publié avec le concours du Centre national de la recherche scientilque 


\title{
INTEGRAL REPRESENTATIONS FOR SOLUTIONS OF EXPONENTIAL GAUSS-MANIN SYSTEMS
}

\author{
By Marco Hien \& CÉline Roucairol
}

\begin{abstract}
Let $f, g: U \rightarrow \mathbb{A}^{1}$ be two regular functions from the smooth affine complex variety $U$ to the affine line. The associated exponential Gauß-Manin systems on the affine line are defined to be the cohomology sheaves of the direct image of the exponential differential system $\mathcal{O}_{U} e^{g}$ with respect to $f$. We prove that its holomorphic solutions admit representations in terms of period integrals over topological chains with possibly closed support and with rapid decay condition.
\end{abstract}

RÉsumé (Représentations intégrales des solutions des systèmes de Gauß-Manin exponentiels)

Soient $f, g: U \rightarrow \mathbb{A}^{1}$ deux fonctions régulières sur une variété affine lisse $U$ à valeurs dans la droite affine. On leurs associe des systèmes de Gauß-Manin sur la droite affine définis comme étant les faisceaux de cohomology de l'image directe par $f$ du système différentiel exponentiel $\mathcal{O}_{U} e^{g}$. Nous prouvons que leurs solutions holomorphes admettent des représentations sous forme d'intégrales de périodes sur des chaînes topologiques à support éventuellement fermé avec une condition de décroissance rapide.

Texte reçu le 5 novembre 2007 et le 7 mars 2008, accepté le 7 juillet 2008

Marco Hien, NWF I - Mathematik, Universität Regensburg, 93040 Regensburg, Germany

- E-mail : marco.hien@mathematik.uni-regensburg.de

CÉline Roucairol, Lehrstuhl für Mathematik VI, Universität Mannheim, A5 6, 68161 Mannheim, Germany - E-mail : celine.roucairol@uni-mannheim.de

2000 Mathematics Subject Classification. - 32S40, 32C38, 14 F40.

Key words and phrases. - Gauß-Manin systems, $\mathcal{D}$-modules. 


\section{Introduction}

Let $U$ be a smooth affine complex variety of dimension $n$ and let $f, g$ : $U \rightarrow \mathbb{A}^{1}$ be two regular functions. We consider the flat algebraic connection $\nabla: \mathcal{O}_{U} \rightarrow \Omega_{U}^{1}$ on the trivial line bundle $\mathcal{O}_{U}$ defined as $\nabla u=d u+d g \cdot u$. Let us denote the associated holonomic $\mathcal{D}_{U}$-module by $\mathcal{O}_{U} e^{g}$, i.e. the trivial $\mathcal{O}_{U}$-module on which any vector field $\xi$ on $U$ acts via the associated derivation $\nabla_{\xi}$ induced by the connection. Let $\mathcal{N}$ be the direct image $\mathcal{N}:=f_{+}\left(\mathcal{O}_{U} e^{g}\right)$ in the theory of $\mathcal{D}$-modules (see e.g. [2]). Then $\mathcal{N}$ is a complex of $\mathcal{D}_{\mathbb{A}^{1}}$-modules and we consider its $k$-th cohomology sheaf

$$
\mathcal{H}^{k} f_{+}\left(\mathcal{O}_{U} e^{g}\right)
$$

a holonomic $\mathcal{D}_{\mathbb{A}^{1}}$-module.

There is an alternative point of view of $\mathcal{H}^{k} \mathcal{N}$ in terms of flat connections: outside a finite subset $\Sigma_{1} \subset \mathbb{A}^{1}$, the $\mathcal{O}_{\mathbb{A}^{1}}$-module $\mathcal{H}^{k} f_{+}\left(\mathcal{O}_{U} e^{g}\right)$ is locally free, hence a flat connection. This connections coincides with the Gauß-Manin connection on the relative de Rham cohomology

$$
\left.\left.\mathcal{H}^{k}(\mathcal{N})\right|_{\mathbb{A}^{1} \backslash \Sigma_{1}} \cong\left(\boldsymbol{R}^{k+n-1} f_{*}\left(\Omega_{U / \mathbb{A}^{1}}^{\cdot}, \nabla\right), \nabla_{G M}\right)\right|_{\mathbb{A}^{1} \backslash \Sigma_{1}},
$$

i.e. the corresponding higher direct image of the complex of relative differential forms on $U$ with respect to $f$ and the differential induced by the absolute connection, as in [6]. It is a flat connection over $\mathbb{A}^{1} \backslash \Sigma_{1}$.

In the case $g=0$, which is classically called the Gauß-Manin system of $f$, it is well-known that the solutions admit integral representations (see [10] and [12]). The aim of the present article is to give such a description of the solutions in the more general case of the Gauß-Manin system of the exponential module $\mathcal{O}_{U} e^{g}$, which we will call exponential Gauß-Manin system. A major difficulty lies in the definition of the integration involved. In the case of vanishing $g$, the connection is regular singular at infinity and the topological cycles over which the integrations are performed can be chosen with compact support inside the affine variety $U$. In the exponential case $g \neq 0$, the connection is irregular singular at infinity and we will have to consider integration paths approaching the irregular locus at infinity.

A systematic examination of period integrals in more general irregular singular situations over complex surfaces has been carried out in [5] resulting in a perfect duality between the de Rham cohomology and some homology groups, the rapid decay homology groups, in terms of period integrals. We will prove in this article that this result generalizes to arbitrary dimension in case of an elementary exponential connection $\mathcal{O} e^{g}$ as before, see Theorem 2.7.

With this tool at hand, we construct a local system $\mathfrak{H}_{k}^{r d}$ on $\mathbb{A}^{1} \backslash \Sigma_{2}$, the stalk at point a $t$ of which is the rapid decay homology $H_{k}^{r d}\left(f^{-1}(t), e^{-g_{t}}\right)$ of the restriction of $\mathcal{O}_{U} e^{-g}$ to the fibre $f^{-1}(t)$. Let $\Sigma=\Sigma_{1} \cup \Sigma_{2}$. We prove that 
given a section $c_{t} \otimes e^{g_{t}}$ in the local system $\mathfrak{H}_{k+n-1}^{r d}$ and a relative differential form $\omega$ in $\Omega_{r e l}^{k+n-1}\left(f^{-1}\left(\mathbb{A}^{1} \backslash \Sigma\right)\right.$ ) (which describes the analytification of $\mathcal{H}^{k} \mathcal{N}$ as in the obvious analytic analogue to Proposition 3.3), the integral

$$
\left.\int_{c_{t}} \omega\right|_{f^{-1}(t)} \cdot e^{g_{t}}
$$

gives a (multi-valued) holomorphic solution of the exponential Gauß-Manin system. As a consequence of the duality proved in Theorem 2.7, we deduce that all (multi-valued) holomorphic solutions on $\mathbb{A}^{1} \backslash \Sigma$ can be obtained by this construction, i.e. we achieve the following theorem (Theorem 3.5) which is the main result of this article:

TheOREm 1.1. - For any simply connected open subset $V \subset \mathbb{C} \backslash \Sigma$, the space of holomorphic solutions of the exponential Gauß-Manin system $\mathcal{H}^{k} \mathcal{N}$ is isomorphic to the space of sections in the local system $\mathfrak{H}_{k+n-1}^{\text {rd }}$ over $V$.

The isomorphism in the theorem is given in terms of the integration (1). We want to remark that this theorem generalizes similar results by F. Pham for the Fourier-Laplace transform of the Gauß-Manin systems of a regular function $h: U \rightarrow \mathbb{A}^{1}$ (consider $f: \mathbb{A}^{1} \times U \rightarrow \mathbb{A}^{1}$ to be the first canonical projection and $g: \mathbb{A}^{1} \times U \rightarrow \mathbb{A}^{1}$ given by $g(x, u)=x h(u)$, cf. [11]). It also includes other well-known examples as the integral representations of the Bessel-functions for instance (cf. the introduction of [1] and the final remark of this article).

\section{The period pairing}

Consider the situation described in the introduction, namely $f, g: U \rightarrow \mathbb{A}^{1}$ being two regular functions on the smooth affine complex variety $U$. The main result of this work is to give a representation of the Gauß-Manin solutions in terms of period integrals. The proof relies on a duality statement between the algebraic de Rham cohomology of the exponential connection associated to $g$ and some Betti homology groups with decay condition. The present section is devoted to the proof of this duality statement, which is a generalization of the analogous result for surfaces in [5] to the case of exponential line bundles in arbitrary dimensions.

2.1. Rapid decay homology. - Let us start with any smooth affine complex variety $U_{t}$ and a regular function $g_{t}: U_{t} \rightarrow \mathbb{A}^{1}$. Note, that the index $t$ is irrelevant in this section but will become meaningful later in the application to the Gauß-Manin system where $U_{t}$ will denote the smooth fibres $f^{-1}(t)$. 
We assume that $U_{t}$ is embedded into a smooth projective variety $X_{t}$ and that $g_{t}$ extends to a meromorphic mapping $g_{t}: X_{t} \rightarrow \mathbb{P}^{1}$. Note, that we do not impose any further conditions on the pole divisor $D_{t}:=g_{t}^{-1}(\infty)$.

Let us denote by $\mathcal{O}_{X_{t}}\left[* D_{t}\right]$ the sheaf of meromorphic functions on $X_{t}$ with poles along $D_{t}$. We will write $\mathcal{O} e^{g_{t}}$ for the flat meromorphic connection

$$
\nabla: \mathcal{O}_{X_{t}}\left[* D_{t}\right] \longrightarrow \Omega_{X_{t} / \mathbb{C}}^{1}\left[* D_{t}\right], u \mapsto d u+u \cdot d g_{t}
$$

on the trivial meromorphic line bundle $\mathcal{O}_{X_{t}}\left[* D_{t}\right]$.

In [5], a duality pairing between the de Rham cohomology of a flat meromorphic connection with poles along a divisor with normal crossings (admitting a good formal structure) and a certain homology theory, the rapid decay homology, is constructed in the case $\operatorname{dim}\left(X_{t}\right)=2$, generalizing previous constructions of Bloch and Esnault for curves. For connections of exponential type lying in the main focus of this work, we will now generalized these results to the case of arbitrary dimension and also weakening the normal crossing assumption on the pole divisor. We remark that a crucial point in [5] is to work with a good compactification with respect to the given vector bundle and connection. For connections of the type $\mathcal{O} e^{g_{t}}$ as above, we can always pull-back to such a good situation by a finite blow-up process.

We now want to define the rapid decay homology of the dual connection $\mathcal{O} e^{-g_{t}}$. Since we do not assume that $D_{t}$ is a normal crossing divisor, we have to modify the definition of [5] for our present purposes. We start with the given meromorphic map $g_{t}: X_{t} \rightarrow \mathbb{P}^{1}$. Consider the real oriented blow-up $\pi: \widetilde{\mathbb{P}^{1}} \rightarrow \mathbb{P}^{1}$ of the point $\infty$ in $\mathbb{P}^{1}$ and let $S_{\infty}^{1}:=\pi^{-1}(\infty)$ be the circle at infinity. We will use the following local presentation of the real oriented blowup of $\infty \in \mathbb{P}^{1}$

$$
\pi^{-1}\left(\mathbb{P}^{1} \backslash 0\right) \cong \mathbb{R}_{0}^{+} \times S^{1} \stackrel{\pi}{\longrightarrow} \mathbb{P}^{1} \backslash 0,\left(r, e^{i \vartheta}\right) \mapsto \frac{1}{r} e^{i \vartheta}
$$

and identify $S_{\infty}^{1}=\pi^{-1}(\infty)$ with $0 \times S^{1}$. We define

$$
\widetilde{X_{t}}=X_{t} \times g_{t} \widetilde{\mathbb{P}^{1}}
$$

as the fibre product of $X_{t}$ and $\widetilde{\mathbb{P}^{1}}$ over $\mathbb{P}^{1}$ and denote the associated maps as in the following diagram:

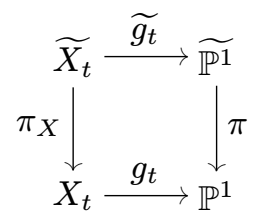

TOME $136-2008-\mathrm{N}^{\mathrm{O}} 4$ 
Restricted to $\pi^{-1}\left(U_{t}\right)$, the map $\pi_{X}$ is a homeomorphism to $U_{t}$ by which we consider $U_{t}$ as an open subset of $\widetilde{X}_{t}$. Let $\tilde{\jmath}: U_{t} \hookrightarrow \widetilde{X}_{t}$ be the embedding. Additionally, let $\mathcal{C}_{\widetilde{X}_{t}}^{-p}$ denote the sheaf associated to the presheaf $V \mapsto S_{p}\left(\widetilde{X_{t}}, \widetilde{X}_{t} \backslash V\right)$ of $\mathbb{Q}$-vector spaces, where $S_{p}(Y)$ denotes the groups of piecewise smooth singular $p$-chains in $Y$ and $S_{p}(Y, A)$ the relative chains for a pair of topological spaces $A \subset Y$.

We adopt the standard sign convention for the resulting complex $\mathcal{C}_{\widetilde{X}_{t}}^{-\cdot}$. i.e. the differential of $\mathcal{C}_{\widetilde{X}_{t}}^{-\cdot}$ will be given by $(-1)^{r}$ times the topological boundary operator $\partial$ on $\mathcal{C}_{\widetilde{X}_{t}}^{-r}$.

Now, let

$$
\mathcal{N}:=\left\{\vartheta:=e^{i \varphi} \in S_{\infty}^{1} \mid \frac{3 \pi}{2} \leq \varphi \leq \frac{5 \pi}{2}\right\} \subset S_{\infty}^{1} \subset \widetilde{\mathbb{P}^{1}}
$$

denote the closed half-circle in $S_{\infty}^{1}$ of non-rapid decay in the sense that the function $r \mapsto \exp \left(\frac{1}{r} \vartheta\right)$ has rapid decay for $r \rightarrow 0$ if and only if $\vartheta \notin \mathcal{N}$.

Consider the subspaces

$$
\mathcal{R}_{t}:=\widetilde{X_{t}} \backslash{\widetilde{g_{t}}}^{-1} \mathcal{N} \subset \widetilde{X_{t}} \quad \text { and } \quad \mathcal{S}_{t}:={\widetilde{g_{t}}}^{-1} S_{\infty}^{1} \cap \mathcal{R}_{t} \subset \widetilde{X_{t}} .
$$

Let $\mathcal{C}_{\mathcal{R}_{t}, \mathcal{S}_{t}}^{-\cdot}$ be the complex of chains in $\mathcal{R}_{t}$ relative to $\mathcal{S}_{t}=\mathcal{R}_{t} \backslash U_{t}$, i.e. the sheaf associated to the presheaf

$$
V \mapsto S .\left(\mathcal{R}_{t},\left(\mathcal{R}_{t} \backslash V\right) \cup \mathcal{S}_{t}\right)
$$

on $\mathcal{R}_{t}$. If $c$ denotes a $p$-chain $c \in \Gamma\left(V, \mathcal{C}_{\mathcal{R}_{t}, \mathcal{S}_{t}}^{-p}\right)$ represented by a map $c: \Delta^{p} \rightarrow \mathcal{R}_{t}$ from the standard $p$-simplex $\Delta^{p}$ to $\mathcal{R}_{t}$ - for simplicity assume that $c$ maps the interior of $\Delta^{p}$ to $U_{t}$ - then the function $\exp \left(g_{t} \circ c(z)\right)$ has rapid decay for $z \in \operatorname{interior}\left(\Delta^{p}\right)$ approaching the boundary $\partial \Delta^{p}$ by the definition of $\mathcal{R}_{t}$.

Definition 2.1. - Consider the inclusions $U_{t} \stackrel{j}{\hookrightarrow} \mathcal{R}_{t} \stackrel{\kappa}{\hookrightarrow} \widetilde{X}_{t}, \kappa$ being a closed embedding, and let $\mathcal{E}$ denote the trivial local system on $U_{t}$ associated to the dual connection $\mathcal{O}_{U_{t}} e^{-g_{t}}$ (spanned by $e^{g_{t}}$ ). The rapid decay complex is defined to be the complex of sheaves

$$
\mathcal{C}_{\widetilde{X}_{t}}^{\mathrm{rd}}\left(e^{-g_{t}}\right):=\kappa_{!}\left(\mathcal{C}_{\mathcal{R}_{t}, \mathcal{S}_{t}}^{-\cdot} \otimes j_{*} \mathcal{E}\right)
$$

on $\widetilde{X}_{t}$. The rapid decay homology of $\mathcal{O} e^{-g_{t}}$ is the corresponding hypercohomology

$$
H_{k}^{r d}\left(U_{t}, e^{-g_{t}}\right):=\mathbb{H}^{-k}\left(\widetilde{X_{t}}, \mathcal{C}_{\widetilde{X}_{t}}^{\text {rd }}\left(e^{-g_{t}}\right)\right)
$$


We will see in Proposition 2.3 that the rapid decay homology is indeed independent of the choice of the compactification $X_{t}$ such that $g_{t}$ admits a meromorphic continuation $g_{t}: X_{t} \rightarrow \mathbb{P}^{1}$.

The usual barycentric subdivision operator on $\mathcal{C}_{\mathcal{R}_{t}, \mathcal{S}_{t}}^{-\cdot}$ induces a subdivision operator on the rapid decay complex. The latter is therefore easily seen to be a homotopically fine complex of sheaves (cp. [18], p. 87). Hence, the rapid decay homology can be computed as the cohomology of the corresponding complex of global sections:

$$
H_{k}^{r d}\left(U_{t}, e^{g_{t}}\right)=H^{-k}\left(\Gamma\left(\widetilde{X_{t}}, \mathcal{C}_{\widetilde{X}_{t}}^{\text {rd }}\left(e^{-g_{t}}\right)\right)\right) .
$$

2.2. The pairing. - We will now define the period pairing in the situation described above. Since $U_{t}$ is assumed to be affine, the de Rham cohomology of $\mathcal{O}_{U_{t}} e^{g_{t}}$ can be computed on the level of global sections

$$
H_{d R}^{p}\left(U_{t}, e^{g_{t}}\right):=\mathbb{H}^{p}\left(U_{t},\left(\Omega_{U_{t} / \mathbb{C}}, \nabla_{t}\right)\right) \cong H^{p}\left(\Gamma\left(U_{t}, \Omega_{U_{t} / \mathbb{C}}^{\cdot}\right), \nabla_{t}\right),
$$

with $\nabla_{t}(\omega)=d \omega+d g_{t} \wedge \omega$ for a local section $\omega$ of $\Omega_{U_{t} / \mathbb{C}}^{q}$.

Now, if we have a global rapid decay chain $c \otimes e^{g_{t}} \in \Gamma\left(\widetilde{X_{t}}, \mathcal{C}_{\widetilde{X}_{t}}^{\text {rd }},-p\left(e^{-g_{t}}\right)\right)$ with respect to the dual bundle $\mathcal{O}_{U_{t}} e^{-g_{t}}$ and a meromorphic $p$-form $\omega$, then the integral $\int_{c} \omega e^{g_{t}}$ converges because the rapid decay of $e^{g_{t}}$ along $c$ annihilates the moderate growth of the meromorphic $\omega$. Let $c_{\tau}$ denote the topological chain one gets by cutting off a small tubular neighborhood with radius $\tau$ around the boundary $\partial \Delta^{p}$ from the given topological chain $c$. Then, for $c \otimes e^{g_{t}} \in$ $\mathcal{C}_{\widetilde{X}_{t}}^{\text {rd }, p}\left(e^{-g_{t}}\right)\left(\widetilde{X}_{t}\right)$ and $\eta \in \Omega^{p-1}\left(* D_{t}\right)$ a meromorphic $(p-1)$-form, we have the "limit Stokes formula"

$$
\int_{c}\left(\nabla_{t} \eta\right) e^{g_{t}}=\lim _{\tau \rightarrow 0} \int_{c_{\tau}}\left(\nabla_{t} \eta\right) e^{g_{t}}=\lim _{\tau \rightarrow 0} \int_{\partial c_{\tau}} \eta e^{g_{t}}=\int_{\partial c-D_{t}} \eta e^{g_{t}}
$$

where in the last step we used that by the given growth/decay conditions the integral over the faces of $\partial c_{\tau}$ 'converging' against the faces of $\partial c$ contained in $D_{t}$ vanishes.

The limit Stokes formula easily shows in the standard way that integrating a closed differential form over a given rd-cycle (i.e. with vanishing boundary value) only depends on the de Rham class of the differential form and the rd-homology class of the cycle. Thus, we have:

Proposition 2.2. - Integration induces a well defined bilinear pairing

$$
H_{d R}^{p}\left(U_{t}, e^{g_{t}}\right) \times H_{p}^{r d}\left(U_{t}, e^{-g_{t}}\right) \longrightarrow \mathbb{C},\left([\omega],\left[c \otimes e^{g_{t}}\right]\right) \mapsto \int_{c} \omega e^{g_{t}},
$$

which we call the period pairing of $\mathcal{O}_{U_{t}} e^{g_{t}}$. 
2.3. Independence of choice of a good compactification. - Assume that we have two compactifications $X_{t}$ and $X_{t}^{\prime}$ of $U_{t}$ such that $g_{t}: U_{t} \rightarrow \mathbb{A}^{1}$ admits meromorphic extensions $g_{t}: X_{t} \rightarrow \mathbb{P}^{1}$ as well as $h_{t}: X_{t}^{\prime} \rightarrow \mathbb{P}^{1}$. Passing to a common compactification (e.g. the closure of $U_{t}$ in $X_{t} \times X_{t}^{\prime}$ ), we can assume that there is a map $b: X_{t}^{\prime} \rightarrow X_{t}$ compatible with $h_{t}$ and $g_{t}$. If $\widetilde{X_{t}}$ and $\widetilde{X_{t}^{\prime}}$ denote the associated spaces as in (3), the compatibility $h_{t}=g_{t} \circ b$ produces a lift $\tilde{b}$

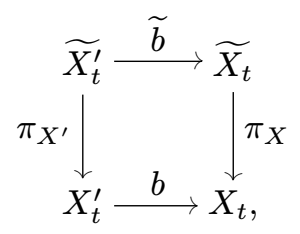

such that $\widetilde{g_{t}} \circ \widetilde{b}=\widetilde{h_{t}}$.

Proposition 2.3. - In the situation above, we have

$$
\mathbb{H}^{-k}\left(\widetilde{X_{t}^{\prime}}, \mathcal{C}_{\widetilde{X}_{t}^{\prime}}^{\text {rd }}\left(e^{-h_{t}}\right)\right)=\mathbb{H}^{-k}\left(\widetilde{X_{t}}, \mathcal{C}_{\widetilde{X}_{t}}^{\text {rd }}\left(e^{-g_{t}}\right)\right) .
$$

Proof. - The proposition follows by standard arguments: We have to consider the subspaces of rapid decay, i.e.

$$
\mathcal{R}_{t}^{\prime}:=\widetilde{X_{t}^{\prime}} \backslash \widetilde{h}_{t}^{-1}(\mathcal{N}) \stackrel{\kappa^{\prime}}{\hookrightarrow} \widetilde{X_{t}^{\prime}} \text { and } \mathcal{R}_{t}:=\widetilde{X_{t}} \backslash \widetilde{g}_{t}^{-1}(\mathcal{N}) \stackrel{\kappa}{\hookrightarrow} \widetilde{X_{t}}
$$

as well as their 'boundaries' $\mathcal{S}_{t}^{\prime}=\mathcal{R}_{t}^{\prime} \backslash U_{t}$ and $\mathcal{S}_{t}=\mathcal{R}_{t} \backslash U_{t}$. Obviously, the lift $\widetilde{b}$ maps $\mathcal{R}_{t}^{\prime}$ to $\mathcal{R}_{t}$, i.e. we have the diagram

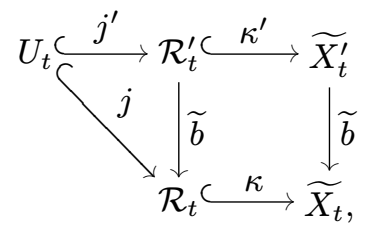

Regarding the definition of the rapid decay complex (6), keeping in mind that the local system $\mathcal{E}=e^{-g_{t}} \mathbb{C}_{U_{t}}$ is trivial, we have to show that

$$
\mathbb{H}^{-k}\left(\widetilde{X_{t}^{\prime}}, \kappa_{!}^{\prime} \mathcal{C}_{\mathcal{R}_{t}^{\prime}, \mathcal{S}_{t}^{\prime}}^{-\cdot}\right) \cong \mathbb{H}^{-k}\left(\widetilde{X_{t}}, \kappa ! \mathcal{C}_{\mathcal{R}_{t}, \mathcal{S}_{t}}^{-\cdot}\right)
$$

The left hand side is equal to $\mathbb{H}^{-k}\left(\widetilde{X}_{t}, \boldsymbol{R} \widetilde{b}_{*} \kappa_{!}^{\prime} \mathcal{C}_{\mathcal{R}_{t}^{\prime}, \mathcal{S}_{t}^{\prime}}^{-}\right)$. Since $\widetilde{b}$ is proper, we know that

$$
\boldsymbol{R} \widetilde{b}_{*} \kappa_{!}^{\prime} \mathcal{C}_{\mathcal{R}_{t}^{\prime}, \mathcal{S}_{t}^{\prime}}^{-}=\kappa_{!} \boldsymbol{R} \widetilde{b}_{*} \mathcal{C}_{\mathcal{R}_{t}^{\prime}, \mathcal{S}_{t}^{\prime}}^{-},
$$

so that it remains to prove that $\boldsymbol{R} \widetilde{b}_{*} \mathcal{C}_{\mathcal{R}_{t}^{\prime}, \mathcal{S}_{t}^{\prime}}^{-}=\mathcal{C}_{\mathcal{R}_{t}, \mathcal{S}_{t}}^{-\cdot}$. Since the complex of sheaves $\mathcal{C}_{\mathcal{R}_{t}^{\prime}, \mathcal{S}_{t}^{\prime}}^{-}$is homotopically fine, we can deduce that $\boldsymbol{R} \widetilde{b}_{*} \mathcal{C}_{\mathcal{R}_{t}^{\prime}, \mathcal{S}_{t}^{\prime}}^{-}=\widetilde{b}_{*} \mathcal{C}_{\mathcal{R}_{t}^{\prime}, \mathcal{S}_{t}^{\prime}}^{-}$ The latter complex is isomorphic to $\mathcal{C}_{\mathcal{R}_{t}, \mathcal{S}_{t}}^{-\cdot}$. To see this, note that the local 
sections $c \in \widetilde{b}_{*} \mathcal{C}_{\mathcal{R}_{t}^{\prime}, \mathcal{S}_{t}^{\prime}}^{-p}$ can be represented by formal linear combinations of maps $\Delta^{p} \rightarrow \mathcal{R}^{\prime}$ from the standard $p$-simplex to $\mathcal{R}^{\prime}$ which map the interior $\operatorname{int}\left(\Delta^{p}\right)$ to $U_{t}$. By continuity, these maps are determined by their restriction to the interior $\operatorname{int}\left(\Delta^{p}\right)$ of the standard simplex. Since $\widetilde{h_{t}}=\widetilde{b} \circ \widetilde{g_{t}}$, the closure of $c\left(\operatorname{int}\left(\Delta^{p}\right)\right)$ in $\widetilde{X_{t}^{\prime}}$ is contained in $\mathcal{R}_{t}^{\prime}$ if and only if the corresponding closure in $\widetilde{X}_{t}$ is contained in $\mathcal{R}_{t}$. This determines the isomorphism between $\widetilde{b}_{*} \mathcal{C}_{\mathcal{R}_{t}^{\prime}, \mathcal{S}_{t}^{\prime}}^{-p}$ and $\mathcal{C}_{\mathcal{R}_{t}, \mathcal{S}_{t}}^{-p}$.

2.4. Comparison with the definition in [5]. - In [5], rapid decay homology was defined for arbitrary meromorphic connections admitting a good formal structure on surfaces in the sense of Sabbah ([17]). One of the main features is to start with a good compactification $X_{t}$ with respect to the given connection $\nabla$ in the sense that $\nabla$ admits a good formal decomposition (see loc. cit.). In particular, the locus at infinity $D_{t}:=X_{t} \backslash U_{t}$ will be a divisor with normal crossings. In our present situation, the simple type of the given connection $\mathcal{O}_{U} e^{g}$ allows to omit the normal crossing condition and additionally to generalize to arbitrary dimension. We now want to prove that the two definitions, namely Definition 2.1 and the one in [5] coincide. This comparison will be important in the proof of the duality of the period pairing in Theorem 2.7.

Let us first recall the definition of [5]. Let $g_{t}: X_{t} \rightarrow \mathbb{P}^{1}$ be a meromorphic extension of $g_{t}: U_{t} \rightarrow \mathbb{A}^{1}$ and assume that $D_{t}:=X_{t} \backslash U_{t}$ is a divisor with normal crossings. Consequently, we can consider the real oriented blow-up $\mathfrak{b}: \mathfrak{X}_{t} \rightarrow X_{t}$ of the components of $D_{t}$ (cp. [8]). Locally at a point $x^{0} \in D_{t}$, we can choose coordinates $x_{1}, \ldots, x_{n}$ such that locally $D_{t}=\left\{x_{1} \cdots x_{k}=0\right\}$ with some $1 \leq k \leq n$. In these coordinates, $\mathfrak{b}$ reads as

$$
\left(\left(r_{\nu}, e^{i \vartheta_{\nu}}\right)_{\nu=1}^{k}, x_{k+1}, \ldots, x_{n}\right) \mapsto\left(r_{1} e^{i \vartheta_{1}}, \ldots, r_{k} e^{i \vartheta_{k}}, x_{k+1}, \ldots, x_{n}\right)
$$

with $r_{\nu} \in \mathbb{R}_{0}^{+}$and $e^{i \vartheta_{\nu}} \in S^{1}$.

Similarly to the construction above, let $\mathcal{C}_{\mathfrak{X}_{t}, \mathfrak{D}_{t}}^{-\cdot}$ be the complex of chains relative to the boundary $\mathfrak{D}_{t}:=\mathfrak{b}^{-1}\left(D_{t}\right)$, i.e. the sheaf associated to

$$
V \mapsto S .\left(\mathfrak{X}_{t},\left(\mathfrak{X}_{t} \backslash V\right) \cup \mathfrak{D}_{t}\right) .
$$

Restricted to $U_{t}, \mathfrak{b}$ is an isomorphism and hence we can consider $U_{t}$ as a subspace of $\mathfrak{X}_{t}$. Let $j: U_{t} \hookrightarrow \mathfrak{X}_{t}$ denote the inclusion.

Let $c \otimes e^{g_{t}} \in \Gamma\left(V, \mathcal{C}_{\mathfrak{X}_{t}, \mathfrak{D}_{t}}^{-} \otimes j_{*} \mathcal{E}\right)$ be a local section over some open $V \subset$ $\mathfrak{X}_{t}$, where we recall that $\mathcal{E}$ denotes the local system associated to the dual connection $\mathcal{O} e^{-g_{t}}$. Assume that $c$ is represented by a piecewise smooth map from the standard $p$-simplex $\Delta^{p}$ to $\mathfrak{X}_{t}$.

Now, consider a point $y \in c\left(\Delta^{p}\right) \cap \mathfrak{D}_{t} \cap V$. Choose local coordinates $x_{1}, \ldots, x_{d}$ of $X_{t}$ around $y=0$ such that $D_{t}=\left\{x_{1} \cdots x_{k}=0\right\}$.

TOME $136-2008-\mathrm{N}^{\mathrm{O}} 4$ 
DeFinition 2.4. - We call $c \otimes e^{g_{t}}$ a rapid decay chain on $\mathfrak{X}_{t}$, if for all $y$ as above, the function $e^{g_{t}(x)}$ has rapid decay for the argument approaching $\mathfrak{D}_{t}$, i.e. if for all $N \in \mathbb{N}^{k}$ there is a $C_{N}>0$ such that

$$
\left|e^{g_{t}(x)}\right| \leq C_{N} \cdot\left|x_{1}\right|^{N_{1}} \cdots\left|x_{k}\right|^{N_{k}}
$$

for all $x \in\left(c\left(\Delta^{p}\right) \backslash \mathfrak{D}_{t}\right) \cap V$ with small $\left|x_{1}\right|, \ldots,\left|x_{k}\right|$.

In case $V \cap \mathfrak{D}_{t}=\varnothing$, we do not impose any condition on $c \otimes e^{g_{t}}$. The subcomplex of $\mathcal{C}_{\mathfrak{X}_{t}, \mathfrak{D}_{t}}^{-\cdot} \otimes j_{*} \mathcal{E}$ consisting of all rapid decay complexes will be denoted by $\mathcal{C}_{\mathfrak{X}_{t}}^{\text {rd }}$.

In this situation with $D_{t}$ a normal crossing divisor, we can now compare the complex $\mathcal{C}_{\mathfrak{X}_{t}}^{\text {rd }}$ on the real oriented blow-up $\mathfrak{X}_{t}$ and the complex $\mathcal{C}_{\widetilde{X}_{t}}^{\text {rd }}$ on the space $\widetilde{X_{t}}=X_{t} \times_{g_{t}} \widetilde{\mathbb{P}^{1}}$ introduced in (2), with $\widetilde{D_{t}}:=\widetilde{X_{t}} \backslash U_{t}$. We first observe that there is a canonical lift of $g$

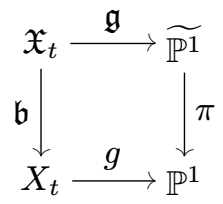

which can be described as follows using the local description above. Consider a point $y \in D_{t}$ and choose coordinates as above such that locally $D_{t}=\left\{x_{1} \cdots x_{k}=0\right\}$ and $y=\left(0, \ldots, 0, x_{k+1}, \ldots, x_{n}\right)$. Let $\xi:=\left(\left(0, e^{i \zeta_{\nu}}\right)_{\nu=1}^{k}, x_{k+1}, \ldots, x_{n}\right)$ be an arbitrary point in $\mathfrak{b}^{-1}(y)$. Then $\mathfrak{g}(\xi)$ is the argument of $g(x)$ for $x \in U_{t}$ approaching $y$ in the direction given by $\left(\zeta_{1}, \ldots, \zeta_{k}\right)$ :

$$
\mathfrak{g}(\xi)=\lim _{r \rightarrow 0} \arg g\left(r e^{i \zeta_{1}}, \ldots, r e^{i \zeta_{k}}, x_{k+1}, \ldots, x_{n}\right) \in S^{1}=\pi^{-1}(\infty) .
$$

Let $\mathfrak{g}: \mathfrak{X}_{t} \rightarrow \widetilde{X_{t}}$ denote the induced mapping also. We now prove the following proposition.

Proposition 2.5. - In the situation above, we have

$$
\boldsymbol{R} \mathfrak{g}_{*} \mathcal{C}_{\mathfrak{X}_{t}}^{\text {rd }}=\mathcal{C}_{X_{t}}^{\text {rd }}
$$

Proof. - The argument is similar to the one in Proposition 2.3. Since both complexes are homotopically fine, we can apply $\mathfrak{g}_{*}$ directly to $\mathcal{C}_{\mathfrak{X}_{t}}^{\text {rd }}$. Let $V \subset \widetilde{X}_{t}$ be some open subset with $V \cap \widetilde{D_{t}} \neq 0$. Now, a piecewise smooth $p$-chain $c: \Delta^{p} \rightarrow \mathfrak{X}_{t}$ which maps the interior int $\left(\Delta^{p}\right)$ to $U_{t}$ induces an element of $\mathfrak{g}_{*} \mathcal{C}_{\mathfrak{X}_{t}}^{\text {rd }}(V)$ if and only if all directions $\xi$ in the closure $c\left(\Delta^{p}\right)$ of $c\left(\operatorname{int}\left(\Delta^{p}\right)\right) \subset \mathfrak{X}_{t}$ are rapid decay directions for $g$, in other words if and only if the implication

$$
\xi \in c\left(\partial \Delta^{p}\right) \Rightarrow \mathfrak{g}(\xi) \in \mathcal{R}_{t}
$$


with the notation $\mathcal{R}_{t}$ from (5) holds. But this is equivalent to saying that $c \otimes e^{g_{t}}$ defines an element of $\mathcal{C}_{\widetilde{X}_{t}}^{\text {rd }}$. Therefore $\mathfrak{g}_{*} \mathcal{C}_{\mathfrak{X}_{t}}^{\text {rd }}=\mathcal{C}_{\widetilde{X}_{t}}^{\text {rd }}$.

Corollary 2.6. - The definitions of rapid decay homology in Definition 2.1 and the one in [5], Definition 2.3-4, coincide.

Proof. - By Proposition 2.3, the rapid decay homology as in Definition 2.1 is independent of the choice of compactification with meromorphic extension of $g_{t}$. Hence, we can choose $X_{t}$ with normal crossing complement and the assertion follows from Proposition 2.5.

2.5. Duality. - We will now prove the following duality theorem for the connection $\mathcal{O}_{U_{t}} e^{g_{t}}$ in arbitrary dimension $\operatorname{dim} U_{t}$.

Theorem 2.7. - The period pairing (8) is a perfect pairing of finite dimensional vector spaces.

The proof given in the following is a generalization of the one in [5] for the special case of a connection $\mathcal{O}_{U_{t}} e^{g_{t}}$ to arbitrary dimension $\operatorname{dim}\left(X_{t}\right) \in \mathbb{N}$.

Proof. - Using resolution of singularities, we can find a compactification $X_{t}$ of $U_{t}$ such that

1. $D_{t}:=X_{t} \backslash U_{t}$ is a divisor with normal crossings and

2. $g_{t}: U_{t} \rightarrow \mathbb{A}^{1}$ admits a meromorphic extension to $X_{t}$.

Let us denote the extension again by $g_{t}: X_{t} \rightarrow \mathbb{P}^{1}$. Due to Corollary 2.6, the rapid decay homology can be computed by the rapid decay complex $\mathcal{C}_{\mathfrak{X}_{t}}^{\text {rd }}$ on the real oriented blow-up $\mathfrak{b}: \mathfrak{X}_{t} \rightarrow X_{t}$ of the local irreducible components of $D_{t}$, i.e. $H_{k}^{r d}\left(U_{t}, e^{-g_{t}}\right)=\mathbb{H}^{-k}\left(\mathfrak{X}_{t}, \mathcal{C}_{\mathfrak{X}_{t}}^{\text {rd }}\right)$.

The proof will rely on a local duality on $\mathfrak{X}_{t}$. To prepare for it, we will describe the period pairing as a local pairing of complexes of sheaves on $\mathfrak{X}_{t}$. We recall the corresponding notions of [5].

Let $\mathcal{A}_{\mathfrak{X}_{t}}^{\bmod D_{t}}$ denote the sheaf of functions on $\mathfrak{X}_{t}$ which are holomorphic on $U_{t}^{\text {an }} \subset \mathfrak{X}_{t}$ and of moderate growth along $\mathfrak{D}_{t}:=\mathfrak{b}^{-1}\left(D_{t}\right)$ and let $\mathcal{A}_{\mathfrak{X}_{t}}^{<D_{t}}$ denote the subsheaf of such functions that are infinitely flat along $\mathfrak{D}_{t}$, i.e. all of whose partial derivatives of arbitrary order vanish on $\mathfrak{D}_{t}$.

We call

$$
\operatorname{DR}_{\mathfrak{X}_{t}}^{\bmod D_{t}}\left(e^{g_{t}}\right):=\mathcal{A}_{\mathfrak{X}_{t}}^{\bmod D_{t}} \otimes_{\mathfrak{b}^{-1}\left(\mathcal{O}_{X_{t}}\right)} \mathfrak{b}^{-1}\left(\mathrm{DR}_{X_{t}^{\operatorname{an}}}\left(\mathcal{O}_{X_{t}}\left[* D_{t}\right] e^{g_{t}}\right)\right)
$$

the moderate de Rham complex of $\mathcal{O}_{U_{t}} e^{g_{t}}$ and

$$
\mathrm{DR}_{\mathfrak{X}_{t}}^{<D_{t}}\left(e^{g_{t}}\right):=\mathcal{A}_{\mathfrak{X}_{t}}^{<D_{t}} \otimes_{\mathfrak{b}-1}\left(\mathcal{O}_{X_{t}}\right) \mathfrak{b}^{-1}\left(\mathrm{DR}_{X_{t}^{\operatorname{an}}}\left(\mathcal{O}_{X_{t}}\left[* D_{t}\right] e^{g_{t}}\right)\right)
$$

TOME $136-2008-\mathrm{N}^{\circ} 4$ 
the asymptotically flat de Rham complex. Note that the first complex computes the meromorphic de Rham cohomology of the meromorphic connection $\mathcal{O}_{X_{t}}\left[* D_{t}\right] e^{g_{t}}$ (cp. [17], Corollaire 1.1.8).

Now, the wedge product obviously defines a pairing of complexes of sheaves on $\mathfrak{X}_{t}$ :

$$
\operatorname{DR}_{\mathfrak{X}_{t}}^{\bmod D_{t}}\left(e^{g_{t}}\right) \otimes_{\mathbb{C}} \operatorname{DR}_{\mathfrak{X}_{t}}^{<D_{t}}\left(e^{-g_{t}}\right) \rightarrow \operatorname{DR}_{\mathfrak{X}_{t}}^{<D_{t}}\left(\mathcal{O}_{X_{t}}, d\right),
$$

where the right hand side denotes the asymptotically flat de Rham complex of the trivial connection. We will compare this pairing to the periods pairing from above.

Before that, let us introduce the sheaf $\mathfrak{D b}_{\mathfrak{X}_{t}}^{\mathrm{rd},-s}$ of rapid decay distributions on $\mathfrak{X}_{t}$, the local section of which are distributions

$$
\varphi \in \mathfrak{D b}_{\mathfrak{X}_{t}}^{-s}(V):=\operatorname{Hom}_{\text {cont }}\left(\Gamma_{c}\left(V, \Omega_{\mathfrak{X}_{t}}^{\infty, s}\right), \mathbb{C}\right)
$$

on the space $\Omega_{\mathfrak{X}_{t}}^{\infty, s}$ of $C^{\infty}$ differential forms on $\mathfrak{X}_{t}$ of degree $s$ with compact support in $\mathfrak{X}_{t}$ satisfying the following condition: we choose coordinates $x_{1}, \ldots, x_{n}$ on $X_{t}$ such that locally on $V$ one has $D_{t}=\left\{x_{1} \cdots x_{k}=0\right\}$. Then we require that for any compact $K \subset V$ and any element $N \in \mathbb{N}^{k}$ there are $m \in \mathbb{N}$ and $C_{K, N}>0$ such that for any test form $\eta$ with compact support in $K$ the estimate

$$
|\varphi(\eta)| \leq C_{K, N} \sum_{i} \sup _{|\alpha| \leq m} \sup _{K}\left\{|x|^{N}\left|\partial^{\alpha} f_{i}\right|\right\}
$$

holds, where $\alpha$ runs over all multi-indices of degree less than or equal to $m$ and $\partial^{\alpha}$ denotes the $\alpha$-fold partial derivative of the coefficient functions $f_{i}$ of $\eta$ in the chosen coordinates.

Integration along chains induces a pairing of complexes of sheaves

$$
\mathrm{DR}_{\mathfrak{X}_{t}}^{\bmod D_{t}, s}\left(e^{g_{t}}\right) \otimes \mathcal{C}_{\mathfrak{X}_{t}}^{\mathrm{rd},-r}\left(e^{-g_{t}}\right) \rightarrow \mathfrak{D} \mathfrak{b}_{\mathfrak{X}_{t}}^{\mathrm{rd}, s-r}
$$

to be defined as follows: for any local $s$-form $\omega$ of $\operatorname{DR}_{\mathfrak{X}_{t}}^{\bmod D_{t}}\left(e^{g_{t}}\right)$, any rapid decay chain $c \otimes e^{g_{t}} \in \Gamma\left(V, \mathcal{C}_{\mathfrak{X}_{t}}^{\text {rd, }-r}\left(e^{-g_{t}}\right)\right)$ and any test form $\eta \in \Gamma_{c}\left(V, \Omega_{\mathfrak{X}_{t}}^{\infty, p}\right)$ with $p=r-s$, the decay and growth assumptions ensure that the integral

$$
\int_{c} \eta \wedge\left(e^{g_{t}} \cdot \omega\right)
$$

converges and moreover, that the distribution that maps $\eta$ to (12) satisfies the condition (10) from above.

Note that $\mathfrak{D} \mathfrak{b}_{\mathfrak{X}_{t}}^{\text {rd,-- }}$ is a fine resolution of $\widetilde{\jmath}_{!} \mathbb{C}_{U_{t}^{\text {an }}}[2 d]$ where $\tilde{\jmath}: U_{t}^{\text {an }} \hookrightarrow \mathfrak{X}_{t}$ denotes the inclusion and $d:=\operatorname{dim}_{\mathbb{C}}\left(X_{t}\right)$. It follows that $\mathbb{H}^{0}\left(\mathfrak{X}_{t}, \mathfrak{D} \mathfrak{b}_{\mathfrak{X}_{t}}^{\text {rd, }}\right) \cong \mathbb{C}$ in a standard way. The resulting pairing $H_{d R}^{p}\left(U_{t}, e^{g_{t}}\right) \times H_{p}^{r d}\left(U_{t}, e^{-g_{t}}\right) \rightarrow \mathbb{C}$ induced by (11) on cohomology in degree zero coincides with the period pairing (Proposition 2.2). 
The proof of Theorem 2.7 now splits into the following intermediate steps (cp. with [5]):

Claim 1. - The complexes $\mathrm{DR}_{\mathfrak{X}_{t}}^{\bmod D_{t}}\left(e^{g_{t}}\right)$ and $\mathrm{DR}_{\mathfrak{X}_{t}}^{<D_{t}}\left(e^{-g_{t}}\right)$ have cohomology in degree zero only.

Proof. - In both cases the argument relies on an existence result of a certain linear partial differential system with moderate or rapidly decaying coefficients. To be more precise, consider the local situation at some point $x_{0} \in D_{t}=$ $\left\{x_{1} \cdots x_{k}=0\right\}$ and let $\vartheta \in \mathfrak{b}^{-1}\left(x_{0}\right) \simeq\left(S^{1}\right)^{k}$ be a direction in $\mathfrak{D}_{t}$ over $x_{0}$. Then the complex of stalks at $\vartheta$ which we have to consider is given as

$$
\cdots \longrightarrow\left(\mathcal{A}_{\mathfrak{X}_{t}}^{? D_{t}} \otimes_{\mathfrak{b}^{-1}} \mathcal{O}_{X_{t}} \mathfrak{b}^{-1} \Omega_{X_{t}}^{p}\right)_{\vartheta} \stackrel{\nabla_{t}}{\longrightarrow}\left(\mathcal{A}_{\mathfrak{X}_{t}}^{? D_{t}} \otimes_{\mathfrak{b}^{-1} \mathcal{O}_{X_{t}}} \mathfrak{b}^{-1} \Omega_{X_{t}}^{p+1}\right)_{\vartheta} \longrightarrow \cdots,
$$

where ? stands for either $<$ or mod. If we describe $\Omega_{X_{t}}^{p}$ in terms of the local basis $d x_{I}$ for all $I=\left\{1 \leq i_{1}<\cdots<i_{p} \leq d\right\}$, the covariant derivation $\nabla_{t}$ in degree $p$ reads as

$$
\sum_{\# I=p} w_{I} d x_{I} \mapsto \sum_{\# J=p+1}\left(\sum_{j \in J} s g n_{J}(j)\left(Q_{j} w_{J \backslash\{j\}}\right)\right) d x_{J}
$$

where

$$
Q_{j} u:=\frac{\partial}{\partial x_{j}} u \pm \frac{\partial g_{t}}{\partial x_{j}} \cdot u,
$$

with the negative sign in case of $e^{g_{t}}$ and the positive in case of $e^{-g_{t}}$. Also, we let $\operatorname{sgn}_{J}(j)=(-1)^{\nu}$ for $J=\left\{j_{1}<\cdots<j_{p+1}\right\}$ and $j_{\nu}=j$.

Now, let $\omega$ be a germ of a section of $\mathcal{A}_{\mathfrak{X}_{t}}^{\text {? }{ }_{t}} \otimes \mathfrak{b}^{-1} \Omega_{X_{t}}^{p+1}$ written in the chosen coordinates as

$$
\omega=\sum_{\#=p+1} w_{J} d x_{J}
$$

such that $\nabla_{t} \omega=0$. We have to find a $p$-form $\eta$ with appropriate growth condition such that $\nabla_{t} \eta=\omega$.

To this end, let $r \in \mathbb{N}$ be an integer such that $w_{J}=0$ for all $J$ with $J \cap\{1, \ldots, r-1\} \neq \varnothing$ (which is an empty condition for $r=1$ ). We prove that we can find a $p$-form $\eta$ with coefficients in $\mathcal{A}_{\mathfrak{X}_{t}}^{\text {? } D_{t}}$ such that

$$
\left(\omega-\nabla_{t} \eta\right) \in \sum_{J \cap\{1, \ldots, r\}=\varnothing} \mathcal{A}_{\mathfrak{X}_{t}, \vartheta}^{? D_{t}} d x_{J} .
$$

Successive application of this argument will prove the claim.

By assumption

$$
0=\nabla_{t} \omega=: \sum_{\# K=p+2}\left(\sum_{k \in K} \operatorname{sgn}_{K}(k)\left(Q_{k} w_{K \backslash\{k\}}\right)\right) d x_{K} .
$$


Taking $k<r$ and $r \in J$ and examining the summand of (15) corresponding to the set $K:=\{s\} \cup J$ we see that

$$
Q_{s} w_{J}=0 \text { for all such } s=1, \ldots, r-1 .
$$

Now, consider the system $\left(\Sigma_{J}\right)$ of partial differential equations for the unknown function $u_{J}$, where $J$ is a fixed subset $J \subset\{r, \ldots, d\}$ of cardinality $p+1$ with $r \in J$ :

$$
\left(\Sigma_{J}\right):\left\{\begin{array}{l}
Q_{s} u_{J}=0 \quad \text { for all } s=1, \ldots, r-1 \\
Q_{r} u_{J}=w_{J},
\end{array}\right.
$$

together with the integrability assumption (16). Systems of this type had been studied by Majima ([8]) before. See for example [16], Appendix A for a presentation from which follows that this system always has a solution in $\mathcal{A}_{\mathfrak{X}_{t}, \vartheta}^{<D_{t}}$ if the coefficients of the system belong to the same space of functions. In the case of moderate growth coefficients/solutions, the assertion follows from [5], Theorem A.1 (which is formulated for dimension 2 only, but generalizes one-to-one to the case of arbitrary dimension).

In each case, we can always find a solution $u_{J} \in \mathcal{A}_{\mathfrak{X}_{t}, \vartheta}^{? D_{t}}$ for any such $J \subset$ $\{r, \ldots, d\}$ of cardinality $p+1$ and $r \in J$ and if we let

$$
\eta:=\sum_{J \text { as above }} u_{J} d x_{J}
$$

we easily see that (14) is satisfied.

Claim 2. - The local pairing (9) is perfect in the derived sense (i.e. the induced morphism

$$
\operatorname{DR}_{\mathfrak{X}_{t}}^{\bmod D_{t}}\left(e^{g_{t}}\right) \rightarrow \boldsymbol{R} \operatorname{Hom}_{\mathfrak{X}_{t}}\left(\mathrm{DR}_{\mathfrak{X}_{t}}^{<D_{t}}\left(e^{-g_{t}}\right), \widetilde{\jmath} ! \mathbb{C}\right)
$$

and the analogous one with $\mathrm{DR}_{\mathfrak{X}_{t}}^{<D_{t}}$ and $\mathrm{DR}_{\mathfrak{X}_{t}}^{\bmod D_{t}}$ exchanged are isomorphisms).

Proof. - According to Claim 1 both complexes have cohomology in degree 0 only, i.e. we are reduced to look at the pairing of sheaves

$$
\mathcal{S}^{\bmod D_{t}} \otimes{ }^{\vee} \mathcal{S}^{<D_{t}} \rightarrow \widetilde{\jmath}_{!} \mathbb{C}_{U_{t}}
$$

with $\mathcal{S}^{\bmod D_{t}}:=\mathcal{H}^{0}\left(\mathrm{DR}_{\mathfrak{X}_{t}}^{\bmod D_{t}}\left(e^{g_{t}}\right)\right)$ and $v^{\vee} \mathcal{S}^{<D_{t}}:=\mathcal{H}^{0}\left(\mathrm{DR}_{\mathfrak{X}_{t}}^{<D_{t}}\left(e^{-g_{t}}\right)\right)$. Again, we consider the local situation $D_{t}=\left\{x_{1} \cdots x_{k}=0\right\}$ and $g_{t}(x)=$ $x_{1}^{-m_{1}} \cdots x_{k}^{-m_{k}} u(x)$, which we restrict to a small enough open polysector $V \subset \mathfrak{X}_{t} \stackrel{\mathfrak{b}}{\rightarrow} X_{t}$. We define the Stokes multi-directions of $g_{t}$ along $D_{t}$ inside $V$ to be

$$
\Sigma_{g_{t}}^{D_{t}}:=S t^{-1}\left(\left(\frac{\pi}{2}, \frac{3 \pi}{2}\right)\right)
$$


where $S t: \mathfrak{D}_{t} \cap V \rightarrow \mathbb{R} / 2 \pi \mathbb{Z}$ denotes the map

$$
S t\left(r_{i}, \vartheta_{i}\right):=-\sum_{i=1}^{k} m_{i} \vartheta_{i}+\arg \left(u \circ \mathfrak{b}\left(r_{i}, \vartheta_{i}\right)\right) .
$$

Let $V_{g_{t}}:=\left(V \backslash \mathfrak{D}_{t}\right) \cup \Sigma_{g_{t}}^{D_{t}}$, i.e. $V_{g_{t}} \cap \mathfrak{D}_{t}$ are the directions in which $e^{g_{t}(x)}$ has rapid decay for $x$ radially approaching $D_{t}$. If $\widetilde{\jmath}_{g_{t}}: V_{g_{t}} \hookrightarrow V$ denotes the inclusion, one obviously has (possibly after shrinking $V$ ):

$$
\left.\mathcal{S}^{\bmod D_{t}}\right|_{V}=\left.\tilde{\jmath}_{-g_{t} !}\left(e^{-g_{t}(x)} \cdot \mathbb{C}_{U_{t}}\right)\right|_{V} \quad \text { and }\left.\quad{ }^{\vee} \mathcal{S}^{<D_{t}}\right|_{V}=\left.\tilde{\jmath}_{g_{t} !}\left(e^{g_{t}(x)} \cdot \mathbb{C}_{U_{t}}\right)\right|_{V}
$$

Consequently we have the following commutative diagram:

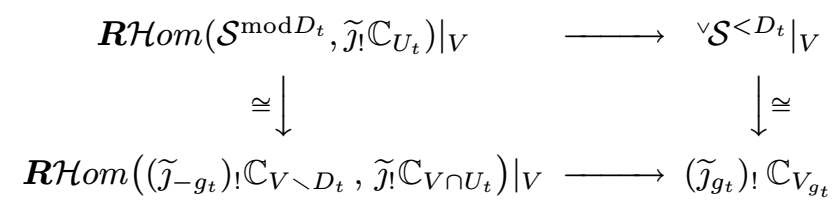

By the factorization $\tilde{\jmath}=\widetilde{\jmath}_{-g_{t}} \circ \widetilde{\iota}_{-g_{t}}$ with $\widetilde{\iota}_{-g_{t}}: V \cap U_{t} \hookrightarrow V_{-g_{t}}$, we see that

$$
\begin{aligned}
& \boldsymbol{R H o m}\left(\left(\widetilde{\jmath}_{-g_{t}}\right) ! \mathbb{C}_{V_{-g_{t}}}, \widetilde{\jmath}_{!} \mathbb{C}_{U_{t}}\right) \cong\left(\widetilde{\jmath}_{-g_{t}}\right)_{*} \boldsymbol{R H} \mathcal{H o m}\left(\mathbb{C}_{V_{-g_{t}}},\left(\widetilde{\iota}_{-g_{t}}\right) ! \mathbb{C}_{V \cap U_{t}}\right) \\
& \cong\left(\widetilde{\jmath}_{-g_{t}}\right)_{*} \mathcal{H o m}\left(\mathbb{C}_{V_{-g_{t}}},\left(\widetilde{\iota}_{-g_{t}}\right) ! \mathbb{C}_{V \cap U_{t}}\right)=\left(\widetilde{\jmath}_{g_{t}}\right) ! \mathbb{C}_{V_{g_{t}}},
\end{aligned}
$$

since $\left(V \backslash V_{g_{t}}\right) \cap \mathfrak{D}_{t}$ coincides with the closure of $V_{-g_{t}} \cap \mathfrak{D}_{t}$ inside $\mathfrak{D}_{t}$. Hence, the bottom line of (18) is an isomorphism and thus

$$
{ }^{\vee} \mathcal{S}^{<D_{t}} \cong \boldsymbol{R} \mathcal{H} \operatorname{lom}_{\mathfrak{X}_{t}}\left(\mathcal{S}^{\bmod D_{t}}, \widetilde{\jmath}_{!} \mathbb{C}_{U_{t}}\right)
$$

locally on $\mathfrak{X}_{t}$ over an arbitrary point of $D_{t}$. Interchanging ${ }^{\vee} \mathcal{S}^{<D_{t}}$ and $\mathcal{S}^{\bmod D_{t}}$ gives the analogous isomorphism.

Claim 3. - There is a canonical isomorphism

$$
\mathcal{C}_{\mathfrak{X}_{t}}^{\mathrm{rd}}\left(e^{-g_{t}}\right) \cong \mathrm{DR}_{\mathfrak{X}_{t}}^{<D_{t}}\left(e^{-g_{t}}\right)[2 d]
$$

in the derived category $D^{b}\left(\mathbb{C}_{\mathfrak{X}_{t}}\right)$, where $d=\operatorname{dim}_{\mathbb{C}}\left(X_{t}\right)$.

Proof. - Here, the arguments of [5], Theorem 3.6, apply directly, we therefore only briefly give the main line of the proof: By Claim 1 we have

$$
\mathrm{DR}_{\mathfrak{X}_{t}}^{<D_{t}}\left(e^{-g_{t}}\right) \stackrel{\sim}{\longleftarrow} \mathcal{H}^{0}\left(\mathrm{DR}_{\mathfrak{X}_{t}}^{<D_{t}}\left(e^{-g_{t}}\right)\right)=:{ }^{\vee} \mathcal{S}^{<D_{t}} .
$$

Additionally, there is a canonical morphism

$$
\mathcal{C}_{\mathfrak{X}_{t}, \mathfrak{D}_{t}}^{-\cdot} \otimes{ }^{\vee} \mathcal{S}^{<D_{t}} \longrightarrow \mathcal{C}_{\mathfrak{X}_{t}}^{\mathrm{rd}}\left(e^{-g_{t}}\right),
$$

since for any open $V \subset \mathfrak{X}_{t}$ and an asymptotically section $\sigma \in \Gamma_{V}\left({ }^{\vee} \mathcal{S}^{<D_{t}}\right)$, the section $\sigma \in \mathcal{V}^{<D_{t}}(V) \subset \widetilde{\jmath}_{*}\left(e^{g_{t}} \mathbb{C}_{U_{t}}\right)$ is rapidly decaying along any chain in $c \in \mathcal{C}_{\mathfrak{X}_{t}, \mathfrak{D}_{t}}^{-\cdot}(V)$, hence $c \otimes \sigma \in \Gamma\left(V, \mathcal{C}_{\mathfrak{X}_{t}}^{\text {rd }}\left(e^{-g_{t}}\right)\right)$. We prove that this morphism is a quasi-isomorphism. Restricted to $U_{t} \subset \mathfrak{X}_{t}$, this is obvious.

TOME $136-2008-\mathrm{N}^{\mathrm{O}} 4$ 
Next, consider a small open polysector $V$ around some $\vartheta \in \mathfrak{b}^{-1}\left(x_{0}\right)$ with $x_{0} \in D_{t}$. Let $\Sigma_{ \pm g_{t}}^{D_{t}} \subset \mathfrak{b}^{-1}\left(x_{0}\right)$ denote the set of Stokes-directions of $e^{ \pm g_{t}}$ as in the proof of Claim 1 .

If $\vartheta \in \Sigma_{-g_{t}}^{D_{t}}$ then for $V$ being a small enough polysector, we have $\left.{ }^{\vee} \mathcal{S}^{<D_{t}}\right|_{V}=$ $\left.\widetilde{\jmath}_{!}\left(e^{g_{t}} \mathbb{C}_{U_{t}}\right)\right|_{V}$. For a smooth topological chain $c$ in $\mathfrak{X}_{t}$, the local section $e^{g_{t}}$ will not have rapid decay along $c$ in $V$ as required by the definition unless the chain does not meet $\mathfrak{D}_{t} \cap V$. Hence

$$
\left.\mathcal{C}_{\mathfrak{X}_{t}}^{\mathrm{rd}}\left(e^{-g_{t}}\right)\right|_{V}=\left.\mathcal{C}_{\mathfrak{X}_{t}, \mathfrak{D}_{t}}^{-\cdot} \otimes{ }^{\vee} \mathcal{S}^{<D_{t}}\right|_{V}
$$

If $\vartheta \in \Sigma_{g_{t}}^{D_{t}}$, we can assume that $V$ is an open polysector such that all the arguments of points in $V$ are contained in $\Sigma_{g_{t}}^{D_{t}}$. Then $\left.{ }^{\vee} \mathcal{S}^{<D_{t}}\right|_{V} \cong \widetilde{\jmath}_{*}\left(e^{g_{t}} \mathbb{C}_{V}\right)$. Similarly, all twisted chains $c \otimes e^{g_{t}}$ will have rapid decay inside $V$ and again both complexes considered are equal to $\mathcal{C}_{\mathfrak{X}_{t}, \mathfrak{D}_{t}}^{-\cdot} \otimes \widetilde{\jmath}_{*}\left(e^{g_{t}} \mathbb{C}_{U_{t}}\right)$.

Finally, if $\vartheta$ separates the Stokes regions of $e^{g_{t}}$ and $e^{-g_{t}}$, we have with the notation of (17):

$$
\left.\left.{ }^{\vee} \mathcal{S}^{<D_{t}}\right|_{V} \cong\left(\widetilde{\jmath}_{g_{t}}\right) !\left(e^{g_{t}} \mathbb{C}_{U_{t}}\right)\right|_{V}
$$

The subspace $V_{g_{t}}$ is characterized by the property that $V_{g_{t}} \cap \mathfrak{D}_{t}$ consists of those directions along which $e^{g_{t}(x)}$ has rapid decay for $x$ approaching $\mathfrak{D}_{t}$. In particular, $c \otimes e^{g_{t}}$ is a rapid decay chain on $V$ if and only if the topological chain $c$ in $\mathfrak{X}_{t}$ approaches $\mathfrak{D}_{t} \cap V$ in $V_{g_{t}}$ at most. Hence

$$
\left.\mathcal{C}_{\mathfrak{X}_{t}}^{\mathrm{rd}}\left(e^{-g_{t}}\right)\right|_{V}=\mathcal{C}_{\mathfrak{X}_{t}, \mathfrak{D}_{t}}^{-\cdot} \otimes\left(\widetilde{\jmath}_{g_{t}}\right) !\left(e^{g_{t}} \mathbb{C}_{U_{t}}\right)=\left.\mathcal{C}_{\mathfrak{X}_{t}, \mathfrak{D}_{t}}^{-\cdot} \otimes{ }^{\vee} \mathcal{S}^{<D_{t}}\right|_{V} .
$$

In summary, we have the following composition of quasi-isomorphisms

$$
{ }^{\vee} \mathcal{S}^{<D_{t}}[2 d] \stackrel{\simeq}{\longrightarrow} \mathbb{C}_{\mathfrak{X}_{t}}[2 d] \otimes \mathcal{S}^{<D_{t}} \stackrel{\simeq}{\longrightarrow} \mathcal{C}_{\mathfrak{X}_{t}, \mathfrak{D}_{t}}^{-\cdot} \otimes{ }^{\vee} \mathcal{S}^{<D_{t}} \stackrel{\simeq}{\longrightarrow} \mathcal{C}_{\mathfrak{X}_{t}}^{\mathrm{rd}}\left(e^{-g_{t}}\right)
$$

In order to prove Theorem 2.7, consider the resolution

$$
\mathcal{A}_{\mathfrak{X}_{t}}^{<D_{t}} \otimes_{\mathfrak{b}^{-1}} \mathcal{O}_{X_{t}} \mathfrak{b}^{-1} \Omega_{X_{t}}^{r} \hookrightarrow\left(\mathcal{P}_{\mathfrak{X}_{t}}^{<D_{t}} \otimes_{\mathfrak{b}^{-1} C_{X_{t}}^{\infty}} \mathfrak{b}^{-1} \Omega_{X_{t}}^{\infty,(r, \bullet)}, \bar{\partial}\right),
$$

where $\mathcal{P}_{\mathfrak{X}_{t}}^{<D_{t}}$ denotes the sheaf of $C^{\infty}$-functions flat at $\mathfrak{b}^{-1}\left(D_{t}\right)$ and $\Omega_{X_{t}}^{\infty,(r, s)}$ denotes the sheaf of $C^{\infty}$ forms on $X_{t}$ of degree $(r, s)$. We then have the bicomplex

$$
\mathcal{R} d^{\bullet, \bullet}:=\left(\mathcal{P}_{\mathfrak{X}_{t}}^{<D_{t}} \otimes_{\mathfrak{b}^{-1} C_{X_{t}}^{\infty}} \mathfrak{b}^{-1} \Omega_{X_{t}}^{\infty,(\bullet, \bullet)}, \partial, \bar{\partial}\right),
$$

whose total complex $\mathcal{R} d^{\bullet}$ computes is a fine resolution of $\widetilde{\jmath}_{!} \mathbb{C}_{U_{t}}$.

According to Claim 1, the local duality pairing (9) reads as $v^{<}{ }^{<D_{t}} \otimes$ $\mathcal{S}^{\bmod D_{t}} \rightarrow \widetilde{\jmath}_{!} \mathbb{C}_{U_{t}^{\text {an }}}$. We now have a canonical quasi-isomorphism

$$
\beta: \mathcal{C}_{\mathfrak{X}_{t}, \mathfrak{D}_{t}}^{-\cdot} \otimes \mathcal{R} d \stackrel{\simeq}{\longrightarrow} \mathfrak{D b}_{\mathfrak{X}_{t}}^{\mathrm{rd},-} \cdot
$$

of complexes mapping an element $c \otimes \rho \in \mathcal{C}_{\mathfrak{X}_{t}, \mathfrak{D}_{t}}^{-r} \otimes \mathcal{R} d^{s}(V)$ of the left hand side over some open $V \subset \mathfrak{X}_{t}$ to the distribution given by $\eta \mapsto \int_{c} \eta \wedge \rho$ for a test form 
$\eta$ with compact support in $V$. The latter represents a local section of $\mathfrak{D} \mathfrak{b}_{\mathfrak{X}_{t}}^{\mathrm{rd}, s-r}$ since $\rho$ is rapidly decaying. Additionally, we have to consider the morphism

$$
\gamma: \mathcal{C}_{\mathfrak{X}_{t}}^{\mathrm{rd}}\left(e^{-g_{t}}\right) \otimes \mathcal{S}^{\bmod D_{t}} \rightarrow \mathfrak{D} \mathfrak{b}_{\mathfrak{X}_{t}}^{\mathrm{rd},-\cdot},\left(c \otimes e^{g_{t}}\right) \otimes \sigma \mapsto\left(\eta \mapsto \int_{c} e^{g_{t}} \cdot \sigma \cdot \eta\right),
$$

which induces the period pairing after taking cohomology.

These morphisms fit into the following commutative diagram

$$
\begin{aligned}
& \vee \mathcal{S}^{<D_{t}}[2 d] \otimes \mathcal{S}^{\bmod D_{t}} \longrightarrow \widetilde{\jmath}_{!} \mathbb{C}_{U_{t}}[2 d] \\
& \simeq \downarrow \simeq \\
& \mathcal{C}_{\mathfrak{X}_{t}, \mathfrak{D}_{t}}^{-\cdot} \otimes{ }^{\vee} \mathcal{S}^{<D_{t}} \otimes \mathcal{S}^{\bmod D_{t}} \longrightarrow \mathcal{C}_{\mathfrak{X}_{t}, \mathfrak{D}_{t}}^{-\cdot} \otimes \mathcal{R} d \cdot \\
& \simeq \text { Claim } 3 \quad \simeq \downarrow \beta \\
& \mathcal{C}_{\mathfrak{X}_{t}}^{\mathrm{rd}}\left(e^{-g_{t}}\right) \otimes \mathcal{S}^{\bmod D_{t}} \stackrel{\gamma}{\longrightarrow} \quad \mathfrak{D b}_{\mathfrak{X}_{t}}^{\mathrm{rd},-\bullet} .
\end{aligned}
$$

The top row is a perfect duality in the derived sense by Claim 2. Applying Poincaré-Verdier duality thus induces an isomorphism

$$
\begin{array}{r}
\boldsymbol{R} \Gamma\left(\mathrm{DR}_{\mathfrak{X}_{t}}^{<D_{t}}\left(e^{-g_{t}}\right)[2 d]\right) \cong \boldsymbol{R} \Gamma \boldsymbol{R} \mathcal{H}_{\mathfrak{X}_{t}}\left(\mathrm{DR}_{\mathfrak{X}_{t}}^{\bmod D_{t}}\left(e^{g_{t}}\right), \widetilde{\jmath}_{!} \mathbb{C}_{U_{t}}[2 d]\right) \cong \\
\cong \operatorname{Hom}_{\mathbb{C}}^{\cdot}\left(\boldsymbol{R} \Gamma\left(\mathrm{DR}_{\mathfrak{X}_{t}}^{\bmod D_{t}}\left(e^{g_{t}}\right)\right), \mathbb{C}\right)
\end{array}
$$

The commutativity of (19) shows that the morphism induced by the period pairing i.e. by $\gamma$, namely

$$
\boldsymbol{R} \Gamma\left(\mathcal{C}_{\mathfrak{X}_{t}}^{\mathrm{rd}}\left(e^{-g_{t}}\right)\right) \longrightarrow \operatorname{Hom}_{\mathbb{C}}^{\cdot}\left(\boldsymbol{R} \Gamma\left(\mathrm{DR}_{\mathfrak{X}_{t}}^{\bmod D_{t}}\left(e^{g_{t}}\right)\right), \mathbb{C}\right),
$$

is an isomorphism. The same holds after exchanging $\mathcal{C}_{\mathfrak{X}_{t}}^{\text {rd }}$ and $\mathrm{DR}_{\mathfrak{X}_{t}}^{\bmod D_{t}}$. Taking $p$-th cohomology therefore yields the perfect period pairing

$$
H_{d R}^{p}\left(U_{t}, e^{g_{t}}\right) \otimes_{\mathbb{C}} H_{p}^{r d}\left(U_{t}, e^{-g_{t}}\right) \rightarrow \mathbb{C}
$$

completing the proof of Theorem 2.7.

We will now study the exponential Gauß-Manin systems and apply these considerations to describe the holomorphic solutions of the latter.

\section{Holomorphic solutions of exponential Gauß-Manin systems}

Consider the situation described in the introduction. Let $f, g: U \rightarrow \mathbb{A}^{1}$ be two regular functions on the smooth affine variety $U$. We denote by $X$ a smooth projective compactification of $U$, on which $f, g: U \rightarrow \mathbb{A}^{1}$ extend to functions $F, G: X \rightarrow \mathbb{P}^{1}$. Let $D:=X \backslash U$ be the complement. 
3.1. Definition. - We define an exponential Gauß-Manin systems as being the cohomology sheaves of the direct image by $f$ of the module of exponential type $\mathcal{O}_{U} e^{g}$ :

$$
\mathcal{G}^{k}:=\mathcal{H}^{k} f_{+}\left(\mathcal{O}_{U} e^{g}\right) .
$$

Let $\Omega_{U}^{k}$ be the sheaf of algebraic differential forms of degree $k$ on $U$. Using the canonical factorization of $f$ into a closed embedding and a projection, we prove as in [4] that:

Proposition 3.1. $-f_{+}\left(\mathcal{O}_{U} e^{g}\right)$ is isomorphic to $f_{*}\left(\Omega_{U}^{\bullet+n} \otimes_{\mathbb{C}} \mathbb{C}\left[\partial_{t}\right]\right)$, where the differential $\nabla$ on $\Omega_{U}^{\bullet+n} \otimes_{\mathbb{C}} \mathbb{C}\left[\partial_{t}\right]$ is given by

$$
\nabla\left(w \otimes \partial_{t}^{i}\right)=d w \otimes \partial_{t}^{i}+d g \wedge w \otimes \partial_{t}^{i}-d f \wedge w \otimes \partial_{t}^{i+1} .
$$

Its cohomology modules $\mathcal{G}^{k}$ are equipped with the structure of a $\mathcal{D}_{\mathbb{A}^{1}}$-module given by:

$$
\begin{aligned}
t\left[w \partial_{t}^{i}\right] & =\left[f w \partial_{t}^{i}-i w \partial_{t}^{i-1}\right], \\
\partial_{t}\left[w \partial_{t}^{i}\right] & =\left[w \partial_{t}^{i+1}\right] .
\end{aligned}
$$

They are holonomic $\mathcal{D}_{\mathbb{A}^{1}}$-modules with possibly irregular singularities on the affine line $\mathbb{A}^{1}$ and at infinity.

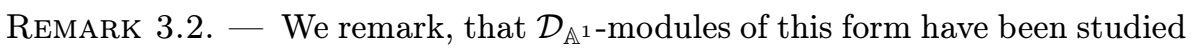
in [14] - [15]. In particular, formulae are given for the irregularities at the singular points as well as for further invariants of the formal connection (in the sense of the Levelt-Turrittin Theorem, see [15] for a precise statement). We will see later, how these results can be applied to deduce information on the growth properties of the period integrals which will appear in our main result, Theorem 3.5.

3.2. The exponential Gauß-Manin systems. - Let $\Sigma_{1}$ be a finite subset of $\mathbb{A}^{1}$ such that, denoting $U_{1}:=f^{-1}\left(\mathbb{A}^{1} \backslash \Sigma_{1}\right)$, we have

i) $f: U_{1} \rightarrow \mathbb{A}^{1} \backslash \Sigma_{1}$ is a locally trivial fibration. For any $t \in \mathbb{A}^{1} \backslash \Sigma_{1}$, the fibre $f^{-1}(t)$ then is a smooth affine variety. We will write $g_{t}$ instead of $\left.g\right|_{f^{-1}(t)}$.

ii) $\left.\mathcal{G}^{k}\right|_{\mathbb{A}^{1} \backslash \Sigma_{1}}$ is locally free, hence a flat connection, for any $k \in \mathbb{Z}$ (recall that $\mathcal{G}^{k}$ is holonomic).

Let $\left(\Omega_{U_{1} /\left(\mathbb{A}^{1} \backslash \Sigma_{1}\right)}^{\bullet+n-1}\left(U_{1}\right), \nabla\right)$ be the complex of relative algebraic differential forms on $U_{1}$ with respect to $f$ equipped with the differential defined by $\nabla(w)=$ $d w+d g \wedge w$.

Proposition 3.3. - The following statements hold: 
1. The space of sections $\Gamma\left(\mathbb{A}^{1} \backslash \Sigma_{1}, \mathcal{G}^{k}\right)$ as a $\mathcal{D}\left(\mathbb{A}^{1} \backslash \Sigma_{1}\right)$-module is isomorphic to $H^{k}\left(\Omega_{U_{1} /\left(\mathbb{A}^{1} \backslash \Sigma_{1}\right)}^{\bullet+n-1}\left(U_{1}\right), \nabla\right)$ with the action of $\mathcal{D}\left(\mathbb{A}^{1} \backslash \Sigma_{1}\right)$ on a form $[w] \in$ $H^{k}\left(\Omega_{U_{1} /\left(\mathbb{A}^{1} \backslash \Sigma_{1}\right)}^{\bullet+n-1}\left(U_{1}\right), \nabla\right)$ given by:

$$
\begin{aligned}
& t \cdot[w]=[f w], \\
& \partial_{t} \cdot[w]=[\eta],
\end{aligned}
$$

where $[\eta] \in H^{k}\left(\Omega_{U_{1} /\left(\mathbb{A}^{1} \backslash \Sigma_{1}\right)}^{\bullet+n-1}\left(U_{1}\right), \nabla\right)$ satisfies $d w+d g \wedge w=d f \wedge \eta$ (which comes from the condition $\nabla(w)=0)$.

2. The sheaf of flat sections of $\left.\mathcal{G}^{k}\right|_{\mathbb{A}^{1} \backslash \Sigma_{1}}$ is a local system on $\mathbb{A}^{1} \backslash \Sigma_{1}$. Its stalk at the point $t$ is given by $H_{d R}^{k+n-1}\left(f^{-1}(t), e^{g_{t}}\right)$.

The local system on $\mathbb{A}^{1} \backslash \Sigma_{1}$ from the second part of the proposition will be denoted by $\mathfrak{H}_{d R}^{k+n-1}$ and we will write $\left(H_{d R}^{k+n-1}, \nabla\right)$ for its associated flat vector bundle.

Proof. - First, we observe that the actions of $t$ and $\partial_{t}$ are well-defined, i.e. do not depend on the choice of the representative $w$. Indeed, if $[w]=0$ we can write $w=d f \wedge \alpha+d \beta+d g \wedge \beta$ with some forms $\alpha$ and $\beta$. Then

$$
t .[w]=[f w]=[d f \wedge(f \alpha-\beta)+d(f \beta)+d g \wedge f \beta]=0 .
$$

Since $d w+d g \wedge w=-d f \wedge(d \alpha+d g \wedge \alpha)$, we furthermore deduce that

$$
\partial_{t} \cdot[w]=-[d \alpha+d g \wedge \alpha]=0 .
$$

To prove the first assertion, recall that $\left.f\right|_{U_{1}}$ is smooth and thus

$$
\left.\mathcal{H}^{k} f_{+}\left(\mathcal{O}_{U} e^{g}\right)\right|_{\mathbb{A}^{1} \backslash \Sigma_{1}}=\boldsymbol{R}^{k} f_{*}\left(\Omega_{U_{1} /\left(\mathbb{A}^{1} \backslash \Sigma_{1}\right)}^{\bullet+n-1}\right)
$$

as left $\mathcal{D}_{\mathbb{A}^{1} \backslash \Sigma_{1}}$-modules, where the $\mathcal{D}$-module structure on the right hand side is induced from the Gauß-Manin connection (cf. Proposition 1.4 in [3]).

Since this a coherent sheaf and $\mathbb{A}^{1} \backslash \Sigma_{1}$ is affine, the usual spectral sequence

$$
H^{i}\left(\mathbb{A}^{1} \backslash \Sigma_{1}, \mathcal{H}^{k-i} \boldsymbol{R} f_{*}\left(\Omega_{U_{1} /\left(\mathbb{A}^{1} \backslash \Sigma_{1}\right)}^{\bullet+n-1}\right)\right) \Longrightarrow \mathbb{H}^{k}\left(\mathbb{A}^{1} \backslash \Sigma_{1}, \boldsymbol{R} f_{*}\left(\Omega_{U_{1} /\left(\mathbb{A}^{1} \backslash \Sigma_{1}\right)}^{\bullet+n-1}\right)\right)
$$

degenerates and we deduce

$$
\begin{aligned}
\Gamma\left(\mathbb{A}^{1} \backslash \Sigma_{1}, \mathcal{G}^{k}\right)=\mathbb{H}^{k}\left(\mathbb{A}^{1} \backslash \Sigma_{1}, \boldsymbol{R} f_{*}\left(\Omega_{U_{1} /\left(\mathbb{A}^{1} \backslash \Sigma_{1}\right)}^{\bullet+n-1}\right)\right)= \\
=\mathbb{H}^{k}\left(U_{1}, \Omega_{U_{1} /\left(\mathbb{A}^{1} \backslash \Sigma_{1}\right)}^{\bullet+n-1}\right)=H^{k}\left(\Omega_{U_{1} /\left(\mathbb{A}^{1} \backslash \Sigma_{1}\right)}^{\bullet+n-1}\left(U_{1}\right), \nabla\right),
\end{aligned}
$$

where the last identification again relies on the coherence of the sheaf under consideration and the fact that $U_{1}$ is affine.

Tоме $136-2008-\mathrm{N}^{\circ} 4$ 
In order to determine the action of $t$ and $\partial_{t}$ on $H^{k}\left(\Omega_{U_{1} /\left(\mathbb{A}^{1} \backslash \Sigma_{1}\right)}^{\bullet+n-1}\left(U_{1}\right), \nabla\right)$, we have to give the previous isomorphism in an explicit way. Similarly to [10] (p.160), one proves that the natural morphism

$$
\begin{aligned}
\Psi: H^{k}\left(\Omega_{U_{1} /\left(\mathbb{A}^{1} \backslash \Sigma_{1}\right)}^{\bullet+n-1}\left(U_{1}\right), \nabla\right) & \left.\rightarrow \Gamma\left(\mathbb{A}^{1} \backslash \Sigma_{1}, \mathcal{G}^{k}\right)\right) \\
{[w] } & \mapsto[-d f \wedge w \otimes 1]
\end{aligned}
$$

is an isomorphism. Note hereby, that $\Psi$ is indeed a morphism of $\mathcal{D}\left(\mathbb{A}^{1} \backslash \Sigma_{1}\right)$ modules since:

- $\Psi(t[w])=\Psi([f w])=[-f d f \wedge w \otimes 1]=t .[-d f \wedge w \otimes 1]=t . \Psi([w])$.

- $\Psi\left(\partial_{t}[w]\right)=\Psi([\eta])=[-d f \wedge \eta \otimes 1]=[-(d w+d g \wedge w) \otimes 1]=\left[-d f \wedge w \otimes \partial_{t}\right]=$ $\partial_{t} \cdot[-d f \wedge w \otimes 1]=\partial_{t} . \Psi([w])$.

As for the second statement of the proposition, we mimic the proof given in [7]. As $\mathcal{G}^{k}$ is a flat connection on $\mathbb{A}^{1} \backslash \Sigma_{1}$, the sheaf of flat sections of $\mathcal{G}^{k}$ is a local system on $\mathbb{A}^{1} \backslash \Sigma_{1}$ and the stalk at the point $t$ is $i_{t}^{+} \mathcal{G}^{k}=\mathcal{H}^{k} i_{t}^{+} f_{+}\left(\mathcal{O}_{U} e^{g}\right)$. Now, according to the base change formula (cf. Theorem 8.4 p. 267 in [2]) and since $\left.f\right|_{U_{1}}$ is smooth,

$$
i_{t}^{+} \mathcal{G}^{k}=\mathcal{H}^{k} f_{t+}\left(\mathcal{O}_{f^{-1}(t)} e^{g_{t}}\right)=H_{d R}^{k+n-1}\left(f^{-1}(t), e^{g_{t}}\right),
$$

where $f_{t}:=\left.f\right|_{f^{-1}(t)}: f^{-1}(t) \rightarrow\{t\}$.

Note that the same statements also hold in the analytic setting, i.e. for

$$
\left(\mathcal{G}^{k}\right)^{\text {an }}:=\mathcal{O}_{\mathbb{C}}^{\text {an }} \otimes_{\mathcal{O}_{\mathbb{A}^{1}}} \mathcal{G}^{k}
$$

with the obvious modifications.

3.3. Family of rapid decay cycles. - We want to apply the period pairing to the fibres of the local system $\left.\mathcal{G}^{k}\right|_{\mathbb{A}^{1} \backslash \Sigma_{1}}$ of Proposition 3.3. To this end, we will now study the corresponding family of rapid decay cycles.

Proposition 3.4. - There exists a finite subset $\Sigma_{2}$ of $\mathbb{A}^{1}$ such that

$$
\bigcup_{t \in \mathbb{A}^{1} \backslash \Sigma_{2}} H_{k}^{r d}\left(f^{-1}(t), e^{-g_{t}}\right) \rightarrow \mathbb{A}^{1} \backslash \Sigma_{2}
$$

is a local system.

We will denote this local system on $\mathbb{A}^{1} \backslash \Sigma_{2}$ by $\mathfrak{H}_{k}^{r d}$. 
Proof. - Let $\mathcal{W}$ be a complex Whitney stratification of $X$ such that $G^{-1}(\infty)$, $D$ and $U$ are union of strata (cf. Theorem (2.2) in [20]). Consider the pull-back $\widetilde{X}$ of the real oriented blow-up $\widetilde{\mathbb{P}^{1}}$ with respect to $G: X \rightarrow \mathbb{P}^{1}$ as in (3):

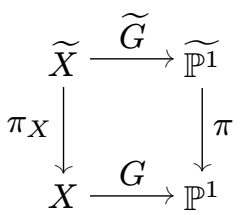

Recall that $S_{\infty}^{1}$ denotes the fibre of $\pi$ over $\infty \in \mathbb{P}^{1}$. With this notation, we have $\pi_{X}^{-1}\left(G^{-1}(\infty)\right)=G^{-1}(\infty) \times S_{\infty}^{1}$.

Let $p \in X$ be any point and let $S_{\alpha}$ be the stratum with $p \in S_{\alpha}$. For the purpose of the proof, we will say that $F$ is $G$-transverse to the stratification $\mathcal{W}$ at $p \in X$, if

$$
T_{F(p)} \mathbb{P}^{1} \oplus 0 \subset \operatorname{image}\left(d(F, G)_{p}: T_{p} S_{\alpha} \longrightarrow T_{F(p)} \mathbb{P}^{1} \oplus T_{G(p)} \mathbb{P}^{1}\right) .
$$

Note that for $p \in S_{\alpha} \subset G^{-1}(\infty)$, the $G$-transversality of $F$ at $p$ reduces to the usual transversality of $F$ with respect to the stratification $\mathcal{W}$.

Now we can proceed as in the first isotopy lemma ([20], Théorèm 4.14). Due to Théorèm 3.3 in loc.cit., there is a Zariski-open dense subset $V \subset(F, G)(X) \subset$ $\mathbb{P}^{1} \times \mathbb{P}^{1}$, such that the restriction of $(F, G)$ to $(F, G)^{-1}(V)$ is transverse to the given stratification $\mathcal{W}$ on $X$. Let $\Delta^{\prime}:=(F, G)(X) \backslash V$ be the complement. Since the stratification respects $G^{-1}(\infty)$, we know that $\mathbb{P}^{1} \times\{\infty\} \subset \Delta^{\prime}$. Therefore, we put $\Delta:=\Delta^{\prime} \backslash\left(\mathbb{P}^{1} \times\{\infty\}\right)$ and consider its closure $\bar{\Delta}$ inside $\mathbb{P}^{1} \times \mathbb{P}^{1}$. Then

$$
\Sigma:=\left\{t_{0} \in \mathbb{A}^{1} \mid\left(t_{0}, \infty\right) \in \bar{\Delta}\right\} \subset \mathbb{A}^{1}
$$

is a finite subset. Additionally, let $\Sigma^{\prime} \subset \mathbb{A}^{1}$ be another finite set, such that $F: X \rightarrow \mathbb{P}^{1}$ is transverse to $\mathcal{W}$ on $F^{-1}\left(\mathbb{A}^{1} \backslash \Sigma^{\prime}\right)$ in the usual sense (again by Théorèm 3.3 in [19]) and let $\Sigma_{2}:=\Sigma \cup \Sigma^{\prime}$.

We conclude, that for any $t_{0} \in \mathbb{A}^{1} \backslash \Sigma$, we can find a small analytic neighbourhood $W$ of $t_{0}$ in $\mathbb{P}^{1}$ such that

1. $\left.F\right|_{F^{-1}(W)}$ is transverse to $\mathcal{W}$ in $F^{-1}(W)$ and

2. in case that $F^{-1}\left(t_{0}\right) \cap G^{-1}(\infty) \neq \varnothing$, there is an analytic neighbourhood $V \subset \mathbb{P}^{1}$ of $\infty$, such that $\left.F\right|_{(F, G)^{-1}(W \times V)}$ is $G$-transverse to $\mathcal{W}$.

In order to achieve the second condition, we just have to choose $W \subset \mathbb{A}^{1}$ and $V \subset \mathbb{P}^{1}$ in such a way that

$$
(W \times V) \cap \bar{\Delta}=\varnothing .
$$

(Note, that the situation in ii) is even better, since we can obtain not only that (21) holds, but even that $d(F, G)_{p}$ is surjective). If $F^{-1}\left(t_{0}\right) \cap G^{-1}(\infty)=\varnothing$, we 
assume that $W$ is small enough such that $F^{-1}(t)$ does not intersect $G^{-1}(\infty)$ for any $t \in W$.

Now we proceed in the standard way ([20], section 4) and construct locally at each $t_{0} \in \mathbb{A}^{1}$ a trivialization of $F$ :

$$
\Phi: F^{-1}(W) \longrightarrow F^{-1}\left(t_{0}\right) \times W
$$

for an open neighbourhood $W$ of $t_{0}$ as above which respects the stratification $\mathcal{W}$. To be more precise: by shrinking we may assume that $W \subset \mathbb{P}^{1}$ is the product of two real intervals $W=I \times J$ with real coordinates $(r, s) \in I \times J$. Proposition (4.6) in [20] allows to lift $\partial / \partial r$ and $\partial / \partial s$ to ruguous vector fields $v_{1}$ and $v_{2}$ on $(X, \mathcal{W})$. Because of the $G$-transversality (condition 2 above), the vector fields $v_{i}$ can be chosen such that

$$
d G_{p} v_{i}(p)=0 \text { for all } p \in F^{-1}(W) \cap G^{-1}(V)
$$

whenever $F^{-1}\left(t_{0}\right) \cap G^{-1}(\infty) \neq \varnothing$ and $W, V$ are chosen as above. Composing the flows of the vector fields $v_{i}$ and possibly shrinking $V$ and $W$, we obtain the trivialization $\Phi: F^{-1}(W) \rightarrow F^{-1}\left(t_{0}\right) \times W$ with the additional condition that in case $F^{-1}\left(t_{0}\right) \cap G^{-1}(\infty) \neq \varnothing$, we have

$$
G \circ \Phi(x, t)=G(x) \text { for all }(x, t) \in\left(F^{-1}\left(t_{0}\right) \cap G^{-1}(V)\right) \times W .
$$

Let us consider the fibre product spaces as in the definition of the rapid decay complex. For given $t_{0} \in \mathbb{A}^{1} \backslash \Sigma_{2}$ let $X_{0}:=F^{-1}\left(t_{0}\right)$ denote the fibre and $G_{0}:=\left.G\right|_{X_{0}}$ the restriction. Then we have

$$
\widehat{X_{0} \times W}:=\left(X_{0} \times W\right) \times_{\left(p r_{1} \circ G_{0}\right)} \widetilde{\mathbb{P}^{1}}=\widetilde{X_{0}} \times W,
$$

where $\widetilde{X_{0}}=X_{0} \times_{G_{0}} \widetilde{\mathbb{P}^{1}}$.

Now, the properties of $\Phi$ discussed above ensure that we can lift $\Phi$ to the these fibre product spaces

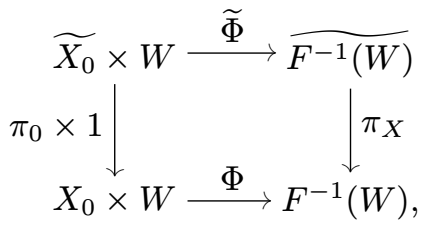

as follows: Outside $G^{-1}(\infty)$, the projections $\pi_{0}: \widetilde{X_{0}} \rightarrow X_{0}$ and $\pi_{X}: \widetilde{X} \rightarrow X$ are homeomorphisms, hence it suffices to consider the situation at $G^{-1}(\infty)$, i.e. we assume that $X_{0} \cap G^{-1}(\infty) \neq \varnothing$ and that we have chosen the open subsets $V \subset \mathbb{A}^{1} \backslash \Sigma_{2}$ and $W \subset \mathbb{P}^{1}$ accordingly. Moreover, it suffices to determine $\widetilde{\Phi}(p, \zeta, t) \in \widetilde{X}$ for

$$
(p, \zeta, t) \in G^{-1}(\infty) \times S_{\infty}^{1} \times W=\pi_{0}^{-1}\left(G^{-1}(\infty)\right) \times W
$$


To this end, consider a sequence of points $\left(x_{n}, t_{n}\right) \in X_{0} \times W$ with $G\left(x_{n}\right) \in$ $V \backslash\{\infty\}$ such that

$$
\lim _{n \rightarrow \infty}\left(x_{n}, t_{n}\right)=(p, t) \in X_{0} \times W \text { and } \lim _{n \rightarrow \infty} G_{0}\left(x_{n}\right)=\zeta \in S_{\infty}^{1},
$$

in other words $\lim _{n \rightarrow \infty}\left(x_{n}, t_{n}\right)=(p, \zeta, t) \in \widetilde{X_{0}} \times W$. By (23), we deduce that

$$
\lim _{n \rightarrow \infty} \arg \left(G \circ \Phi\left(x_{n}, t_{n}\right)\right)=\zeta \in S_{\infty}^{1}
$$

and hence $\lim _{n \rightarrow \infty} \Phi\left(x_{n}, t_{n}\right)=(\Phi(p, t), \zeta) \in G^{-1}(\infty) \times S_{\infty}^{1}$ in the topology of $\widetilde{X}$. In consequence, defining

$$
\widetilde{\Phi}(p, \zeta, t):=(\Phi(p, t), \zeta)
$$

gives a continuous lift $\widetilde{\Phi}$ as desired. It is moreover clear that this lift respects the rapid decay directions along $G^{-1}(\infty)$, i.e. if $\mathcal{R}_{t}=\widetilde{X}_{t}$ denotes the rapid decay subspace inside the fibre $\widetilde{X}_{t}:=\left(F \circ \pi_{X}\right)^{-1}(t)$ as in (5) - namely $\mathcal{R}_{t}:=$ $\widetilde{X}_{t} \backslash{\widetilde{G_{t}}}^{-1}(\mathcal{N})$ with $\mathcal{N}:=\left[\frac{3 \pi}{2}, \frac{5 \pi}{2}\right] \subset S_{\infty}^{1}-$ we have

$$
\widetilde{\Phi}\left(\mathcal{R}_{0} \times\{t\}\right)=\mathcal{R}_{t} \subset \widetilde{X_{t}} \text { for all } t \in W,
$$

where $\mathcal{R}_{0}:=\mathcal{R}_{t_{0}}$.

It follows that $\widetilde{\Phi}$ induces a map

$$
T: \bigcup_{t \in \mathbb{A}^{1} \backslash \Sigma_{2}} H_{k}^{r d}\left(U_{t}, e^{-g_{t}}\right) \longrightarrow H_{k}^{r d}\left(U_{0}, e^{-g_{0}}\right) \times W
$$

given by $T\left(c_{t} \otimes e^{g_{t}}\right)=\left(\left(\operatorname{pr}_{1} \circ \widetilde{\Phi}^{-1} \circ c_{t}\right) \otimes e^{g_{0}}, t\right)$ with $\operatorname{pr}_{1}: X_{0} \times W \rightarrow X_{0}$ the projection. By shrinking, we can assume that $W \subset \mathbb{P}^{1}$ is contractible. Then the canonical inclusion induces an isomorphism

$$
i_{t}: H_{k}^{r d}\left(U_{t}, e^{-g_{t}}\right) \longrightarrow H_{k}^{r d}\left(F^{-1}(W), e^{-g}\right)
$$

and the restriction $T_{t t_{0}}: H_{k}^{r d}\left(U_{t}, e^{-g_{t}}\right) \rightarrow H_{k}^{r d}\left(U_{0}, e^{-g_{0}}\right)$ fits into the commutative diagram

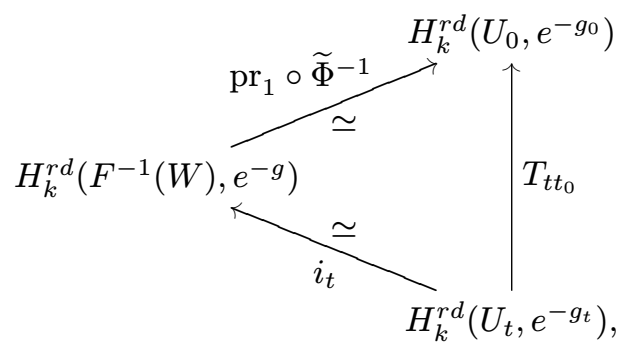

which proves that $T_{t t_{0}}$ is an isomorphism. Moreover, a different choice for $\Phi$ leads to constant transition maps between the corresponding trivializations $T$ and hence $\bigcup_{t \in \mathbb{A}^{1} \backslash \Sigma_{2}} H_{k}^{r d}\left(U_{t}, e^{-g_{t}}\right) \rightarrow \mathbb{A}^{1} \backslash \Sigma_{2}$ is indeed a local system.

TOME $136-2008-\mathrm{N}^{\mathrm{O}} 4$ 
3.4. Holomorphic solutions of the exponential Gauss-Manin systems. - We want to describe the holomorphic solutions of $\mathcal{G}^{k}$, i.e. the morphisms of $\mathcal{D}_{\mathbb{C}}^{\text {an }}$-modules $\left(\mathcal{G}^{k}\right)^{\text {an }} \rightarrow \mathcal{O}_{\mathbb{C}}^{\text {an }}$.

Let $\Sigma=\Sigma_{1} \cup \Sigma_{2}$. We recall that $\left(\mathcal{G}^{k}\right)^{\text {an }}(V)$ is isomorphic to

$$
\mathcal{O}^{\text {an }}(V) \otimes_{\mathbb{C}[t]} H^{k}\left(\Omega_{U / \mathbb{A}^{1}}^{\bullet+n-1}\left(f^{-1}\left(\mathbb{A}^{1} \backslash \Sigma\right)\right), \nabla\right)
$$

for any open subset $V$ in $\mathbb{A}^{1} \backslash \Sigma$ (cf. Proposition 3.3) and $\mathfrak{H}_{k+n-1}^{r d}(V)$ is the space of families on $V$ of cycles in $H_{k+n-1}^{r d}\left(f^{-1}(t), e^{-g_{t}}\right)$ which depend continuously on $t$ (Proposition 3.4).

THEOREM 3.5. - Let $V$ be a simply connected open subset of $\mathbb{A}^{1} \backslash \Sigma$. The morphism

$$
\begin{aligned}
\Psi: \mathfrak{H}_{k+n-1}^{r d}(V) & \rightarrow \operatorname{Hom}_{\mathcal{D}^{\mathrm{an}}(V)}\left(\left(\mathcal{G}^{k}\right)^{\operatorname{an}}(V), \mathcal{O}^{\mathrm{an}}(V)\right) \\
\left(c_{t} \otimes e^{g_{t}}\right)_{t \in V} & \mapsto\left(a \otimes[w] \mapsto\left(I: t \mapsto \int_{c_{t}} a(t) w_{\mid f^{-1}(t)} e^{g_{t}}\right)\right)
\end{aligned}
$$

is a well-defined isomorphism.

In other words, the space of holomorphic solutions of $\mathcal{G}^{k}$ on $V$ is isomorphic to $\mathfrak{H}_{k+n-1}^{r d}(V)$.

Proof. - Since $\nabla_{t}\left(a(t) w_{\mid f^{-1}(t)}\right)=a(t) \nabla(w)$ and $\int \cdot e^{g_{t}}$ defines a pairing between $H_{k+n-1}^{r d}\left(f^{-1}(t), e^{-g_{t}}\right)$ and $H_{d R}^{k+n-1}\left(f^{-1}(t), e^{g_{t}}\right)$ (cf. Proposition 2.2 for $\left.U_{t}=f^{-1}(t)\right), I(t)$ is well-defined for any $t \in V$.

Next, we prove that $I$ is holomorphic on $V$ and that we have constructed a morphism of $\mathcal{D}^{\text {an }}(V)$-modules. Let $t_{0} \in V$. We consider a small contractible neighbourhood $W \subset \mathbb{A}^{1} \backslash \Sigma$ of $t_{0}$ as in the proof of Proposition 3.4 and let $\Phi$ denote the trivialization

$$
\Phi: F^{-1}(W) \longrightarrow F^{-1}\left(t_{0}\right) \times W
$$

we constructed in (22). We denote by $\phi$ the restriction of $\Phi$ to $f^{-1}\left(t_{0}\right) \times W$, which is a homeomorphism of $f^{-1}\left(t_{0}\right) \times W$ to $f^{-1}(W)=U \cap F^{-1}(W)$. For any $t \in W$, we have

$$
I(t)-I\left(t_{0}\right)=\int_{c_{t}} a(t) w_{\mid f^{-1}(t)} e^{g_{t}}-\int_{c_{t_{0}}} a\left(t_{0}\right) w_{\mid f^{-1}\left(t_{0}\right)} e^{g_{t_{0}}}=\int_{c_{t}-c_{t_{0}}}(a \circ f) w e^{g} .
$$

Let $C=\underset{s \in\left[t_{0}, t\right]}{\cup} c_{s}$. Then $\partial C=c_{t}-c_{t_{0}}$ and according to the Stokes' Formula

$$
I(t)-I\left(t_{0}\right)=\int_{C} d\left((a \circ f) w e^{g}\right) .
$$


But since $\nabla([w])=0$, there exists $\eta \in \Gamma_{f^{-1}\left(\mathbb{A}^{1} \backslash \Sigma\right)}\left(\Omega_{U}^{k+n-1}\right)$ such that $d w+$ $d g \wedge w=d f \wedge \eta$. Then,

$$
\begin{aligned}
d\left((a \circ f) w e^{g}\right)=\left(d f \wedge\left(\left(\frac{\partial a}{\partial t} \circ f\right) w\right)\right. & +(a \circ f)(d w+d g \wedge w)) e^{g}= \\
& =d f \wedge\left(\left(\frac{\partial a}{\partial t} \circ f\right) w+(a \circ f) \eta\right) e^{g}
\end{aligned}
$$

and by Fubini's Theorem,

$$
I(t)-I\left(t_{0}\right)=\int_{t_{0}}^{t}\left(\int_{c_{s}}\left(\left(\frac{\partial a}{\partial t} \circ f\right) w+(a \circ f) \eta\right) e^{g}\right) d s .
$$

With $\alpha:=\left(\frac{\partial a}{\partial t} \circ f\right) w+(a \circ f) \eta$, we have $\int_{c_{s}} \alpha e^{g}=\int_{c_{t_{0}}} \widetilde{\phi}^{*}\left(\alpha e^{g_{t}}\right)$.

According to the construction of $\Phi$ and the property (23), we conclude that there exists a compact subset $K$ in $U$ such that $\phi^{*}\left(\alpha e^{g_{t}}\right)=\phi^{*}(\alpha) e^{g_{t_{0}}}$ on $c_{t_{0}} \backslash K$. Now, since $\phi^{*}\left(\alpha e^{g_{t}}\right)$ is continuous in $s$ and $K$ is compact, $\int_{c_{t_{0}} \cap K} \phi^{*}\left(\alpha e^{g_{t}}\right)$ is also continuous in $s$. Furthermore, since $e^{g_{t_{0}}}$ does not depend on $s$ and has rapid decay on $c_{t_{0}} \backslash K$, we deduce that

$$
\int_{c_{t_{0}} \backslash K} \phi^{*}\left(\alpha e^{g_{t}}\right)=\int_{c_{t_{0}} \backslash K} \phi^{*}(\alpha) e^{g_{t_{0}}}
$$

is continuous in $s$ as well.

We conclude that $\int_{c_{t_{0}}} \phi^{*}\left(\alpha e^{g_{t}}\right)$ is continuous in $s$ and hence

$$
\lim _{t \rightarrow t_{0}} \frac{I(t)-I\left(t_{0}\right)}{t-t_{0}}=\int_{c_{t_{0}}} \alpha e^{g}
$$

proving the holomorphicity of $I$ on $V$.

Moreover, since $\partial_{t}(a \otimes[w])=\frac{\partial a}{\partial t} \otimes[w]+a \otimes[\eta]$, the morphism

$$
\left(a \otimes[w] \mapsto\left(I: t \mapsto \int_{c_{t}} a(t) w_{\mid f^{-1}(t)} e^{g_{t}}\right)\right)
$$

is a morphism of $\mathcal{D}^{\text {an }}(V)$-modules.

Finally, we have to prove that $\Psi$ is an isomorphism. According to Theorem 2.7, we have an isomorphism

$$
\begin{aligned}
H_{k+n-1}^{r d}\left(f^{-1}(t), e^{-g_{t}}\right) & \rightarrow \operatorname{Hom}_{\mathbb{C}}\left(H_{d R}^{k+n-1}\left(f^{-1}(t), e^{g_{t}}\right), \mathbb{C}\right) \\
c_{t} \otimes e^{g_{t}} & \mapsto\left(w_{t} \mapsto \int_{c_{t}} w_{t} e^{g_{t}}\right),
\end{aligned}
$$

and consequently $\mathfrak{H}_{k+n-1}^{r d}(V) \simeq \operatorname{Hom}_{\mathbb{C}}\left(\mathfrak{H}_{d R}^{k+n-1}(V), \mathbb{C}\right)$.

Finally, via the isomorphism

$$
\operatorname{Hom}_{\mathbb{C}}\left(\mathfrak{H}_{d R}^{k+n-1}(V), \mathbb{C}\right) \simeq \operatorname{Hom}_{\mathcal{D}^{\text {an }}(V)}\left(\left(\mathcal{G}^{k}\right)^{\text {an }}(V), \mathcal{O}^{\text {an }}(V)\right)
$$

(cf. Corollary 7.1 .1 p. 71 of [10]), we deduce that the morphism $\Psi$ is indeed an isomorphism and the theorem is proved.

TOME $136-2008-\mathrm{N}^{\mathrm{O}} 4$ 
We want to conclude with a view towards an application of this theorem together with previous results of the second named author in [15]. Let $\mathcal{M}$ denote a meromorphic connection on the projective line with singularities in a finite set $\Sigma$. We consider the local situation at a singularity $p \in \Sigma$ and choose a coordinate $t$ such that $p=0$. Let $\widehat{\mathcal{M}}_{0}$ denote the formal completion of $\mathcal{M}$ at 0. Then, according to the Levelt-Turrittin Theorem (cf. [9], Théorème III.1.2) one finds a ramification map $\rho: x \mapsto x^{q}=t$ for some exponent $q$ such that the pull-back $\rho^{-1} \widehat{\mathcal{M}}_{0}$ decomposes into a direct sum

$$
\rho^{-1} \widehat{\mathcal{M}}_{0}=\bigoplus_{i=1}^{r}\left(e^{g_{i}} \otimes R_{i}\right)^{\wedge},
$$

where $e^{g_{i}}:=\mathcal{O}_{\mathbb{P}^{1}, 0} e^{g_{i}}$ denotes the germ of the rank one connection associated to $g_{i} \in \mathcal{O}_{\mathbb{P}^{1}}(* 0)_{0}=\mathbb{C}\{x\}\left[x^{-1}\right]$ as before, $R_{i}$ is a regular singular connection and $(.)^{\wedge}$ denotes formal completion.

In the same situation $\mathcal{M}=\left.\mathcal{H}^{k} f_{+}\left(\mathcal{O}_{U} e^{g}\right)\right|_{\mathbb{A}^{1} \backslash \Sigma}$ as considered in this article, the second named author achieves formulae for the irregularity ([14] and [13]) of $\mathcal{M}$ at $p$ and also for the ramification exponent $q$, the exponents $g_{i}$ in the Levelt-Turrittin decomposition (24) and the rank of the regular factors $R_{i}$ (and some further information on the monodromy of $R_{i}$ ) (cf. [15]) in terms of the given data.

On the other hand side, we know that these informations on the formal structure (24) suffice to determine the asymptotic behaviour of the solutions of the meromorphic connection in small sectors at the singularity (cf. [9], IV.2). Due to Theorem 3.5, we can express the solutions in terms of period integrals and the formulae in [15] and the reasoning just given provides a priori information on the asymptotic behaviour of these period integrals.

Let us illustrate this with a classic example by examining the Bessel equation from this point of view. We start with $U=\mathbb{G}_{m} \times \mathbb{G}_{m}$ compactified by $X=$ $\mathbb{P}^{1} \times \mathbb{P}^{1}$ and coordinates $(u, z)$. Consider the connection $\mathcal{O} e^{g}$ with $g(u, z):=$ $\frac{1}{2} t\left(u-\frac{1}{u}\right)$ and let $f: U \rightarrow \mathbb{G}_{m}$ be the projection $(u, t) \mapsto t$. For any $t$, the restriction to the fibre $X_{t}=\mathbb{P}^{1}$ is irregular singular at $u=0$ and at $u=\infty$, in both cases with irregularity one.

The first relative de Rham cohomology $\mathcal{G}^{0}\left(\mathbb{G}_{m}\right)=H^{1}\left(\Omega_{U / \mathbb{G}_{m}}^{\cdot}(U)\right)$ is a twodimensional vector space with a basis $\left[u^{-n-2} d u\right],\left[u^{-n-1} d u\right]$ for some fixed $n \in \mathbb{Z}$, which can be easily seen from the relation $\left[u^{k} d u\right]=-\frac{2 k}{t}\left[u^{k-1} d u\right]-$ $\left[u^{k-2} d u\right] \in \mathcal{G}^{0}\left(\mathbb{G}_{m}\right)$ for $k \in \mathbb{Z}$. The $\mathcal{D}$-module structure on $\mathcal{G}^{0}$ is induced by the Gauß-Manin connection

$$
\nabla_{G M}\left(u^{k} d u\right)=\frac{1}{2}\left(u^{k+1}-u^{k-1}\right) d u \otimes d t .
$$


We use the basis $\left[u^{-n-2} d u\right],\left[u^{-n-1} d u\right]$ as a trivialization of the Gauß-Manin system which then reads as

$$
\nabla_{G M}=d+\left(\begin{array}{cc}
-\frac{n+1}{t} & -1 \\
1 & \frac{n}{t}
\end{array}\right) d t
$$

Now $S:=\left(\begin{array}{cc}t & 0 \\ -n & t\end{array}\right)$ transforms this system into the standard system associated to the classical Bessel differential equation

$$
\left(t^{2} \frac{d^{2}}{d t^{2}}+t \frac{d}{d t}+\left(t^{2}-n^{2}\right)\right) y=0
$$

which is regular singular at $t=0$ and irregular singular at $t=\infty$ of irregularity one. Note that the usual correspondence between the flat sections of a connection and the $\mathcal{D}$-linear morphisms from the associated $\mathcal{D}$-modules to the function space considered involves a transition to the dual connection. In order to obtain analytic solutions of (25), we therefore apply Theorem 3.5 to the dual connection $\mathcal{O} e^{-g}$.

A local trivialization of the rapid decay local system (see Proposition 3.4) is given by the choice of basis $c_{1} \otimes \varepsilon$ and $c_{2} \otimes \varepsilon$ where $\varepsilon:=e^{\frac{1}{2} t\left(u-\frac{1}{u}\right)}$ is a generator of the local system $\mathcal{E}$ of $\mathcal{O} e^{g}, c_{1}=\{|u|=1\}$ is a loop around the singular point $u=0 \in X_{t}$ and $c_{2}$ is any path in $X_{t}=\mathbb{G}_{m}$ from $u=0$ to $u=\infty$ which fulfills the condition that locally at $u=0$ as well as at $u=\infty$ it is contained in some sector such that $\operatorname{Re}\left(t\left(u-\frac{1}{u}\right)\right)<0$ for all small $u$ inside this sector, i.e. that $\varepsilon$ is rapidly decaying as $u$ moves into $u=0$ or $u=\infty$ respectively along $c_{2}$. Note that $c_{1} \otimes \varepsilon$ is a generator of the usual singular homology group $H_{1}\left(X_{t}, \mathcal{E}\right)$, whereas $c_{2} \otimes \varepsilon$ arises from the fact that 0 and $\infty$ are irregular singular points of the connection on $X_{t}$.

The holomorphic solutions of the Bessel equation (25) our theorem gives (after applying the transformation $S$ and transition to the second order equation) are the period integrals

$$
\int_{c_{i}} e^{\frac{1}{2} t\left(u-\frac{1}{u}\right)} u^{-n-1} d u \text { for } i=1,2 .
$$

Now, the formal structure of the Gauß-Manin system $\mathcal{M}:=\mathcal{G}^{0}$ at $t=\infty$ can either be determined with the methods of [15] from the given data or by following the proof of the Levelt-Turrittin theorem in [9], III.1.2, starting with the differential operator of (25), and one easily obtains that

$$
\widehat{\mathcal{M}}_{\infty}=\left(e^{i t} \otimes R_{1} \oplus e^{-i t} \otimes R_{2}\right)^{\wedge}=:\left(\mathcal{M}^{e l}\right)^{\wedge},
$$

where $R_{i}$ are germs of regular singular rank one connections at infinity. The analytic solutions of the elementary model on the right hand side are of the form 
$e^{i t} \alpha(t)$ and $e^{-i t} \beta(t)$ where $\alpha, \beta$ are solutions of regular singular connections and hence of moderate growth at infinity. Now, given a fixed direction $\vartheta \in S^{1}$ at $t=\infty$, this isomorphism can be lifted to an analytic isomorphism in a small sector around the direction $\vartheta$. More precisely, if $\mathcal{A}_{\vartheta}$ denotes the space of germs of holomorphic functions on small open sectors around the direction $\vartheta$ which admit an asymptotic expansion (see [9], p. 53), viewed as a $K$-algebra where $K=\mathbb{C}\left\{t^{-1}\right\}[t]$ is the field of germs of meromorphic functions at $t=\infty$, there exists a lift

$$
\lambda_{\vartheta}: \mathcal{A}_{\vartheta} \otimes_{K} \mathcal{M}_{\infty} \stackrel{\cong}{\longrightarrow} \mathcal{A}_{\vartheta} \otimes_{K} \mathcal{M}^{e l}
$$

of the decomposition (27) over $\mathcal{A}_{\vartheta}$ (see [9], IV.1-2 for details) and hence the analytic solutions of $\left.\mathcal{M}\right|_{\mathbb{G}_{m}}$ in such a sector, in particular the period integrals (26), can be written in a form $a(t) e^{i t}+b(t) e^{-i t}$ with sections $a, b$ representing germs in $\mathcal{A}_{\vartheta} \otimes \mathcal{M}$. In this sense, the growth properties of the period integrals (26) is governed by the growth properties of $e^{i t}$ and $e^{-i t}$.

\section{Acknowledgments}

We thank the anonymous referee for various substantial suggestions which led to a significant improvement of the above presentation.

\section{BIBLIOGRAPHY}

[1] S. Bloch \& H. Esnault - "Homology for irregular connections", J. Théor. Nombres Bordeaux 16 (2004), p. 357-371.

[2] A. Borel - Algebraic D-modules, Perspectives in Math., vol. 2, Academic Press, 1987.

[3] A. Dimca, F. MaAref, C. Sabbah \& M. Saito - "Dwork cohomology and algebraic D-modules", Math. Ann. 318 (2000), p. 107-125.

[4] A. Dimca \& M. SAito - "On the cohomology of a general fiber of a polynomial map", Compositio Math. 85 (1993), p. 299-309.

[5] M. HIEN - "Periods for irregular singular connections on surfaces", Math. Ann. 337 (2007), p. 631-669.

[6] N. M. KATZ - "On the calculation of some differential Galois groups", Invent. Math. 87 (1987), p. 13-61.

[7] F. MAAREF - "Sur un analogue irrégulier de la connexion de GaussManin", Ann. Fac. Sci. Toulouse Math. (6) 8 (1999), p. 117-124.

[8] H. MAJima - Asymptotic analysis for integrable connections with irregular singular points, Lecture Notes in Mathematics, vol. 1075, Springer, 1984.

[9] B. Malgrange - Équations différentielles à coefficients polynomiaux, Progress in Mathematics, vol. 96, Birkhäuser, 1991. 
[10] F. Pнам - Singularités des systèmes différentiels de Gauss-Manin, Progress in Mathematics, vol. 2, Birkhäuser, 1979.

[11] _ "La descente des cols par les onglets de Lefschetz, avec vues sur Gauss-Manin", Astérisque 130 (1985), p. 11-47.

[12] _ Intégrales singulières, Savoirs Actuels, EDP Sciences/CNRS Éditions, 2005.

[13] C. Roucairol - "Irregularity of an analogue of the Gauss-Manin systems", Bull. Soc. Math. France 134 (2006), p. 269-286.

[14] _ "The irregularity of the direct image of some $\mathcal{D}$-modules", Publ. Res. Inst. Math. Sci. 42 (2006), p. 923-932.

[15] _ "Formal structure of direct image of holonomic $\mathcal{D}$-modules of exponential type", Manuscripta Math. 124 (2007), p. 299-318.

[16] C. SABBAH - "Équations différentielles à points singuliers irréguliers en dimension 2", Ann. Inst. Fourier (Grenoble) 43 (1993), p. 1619-1688.

[17] _ "Équations différentielles à points singuliers irréguliers et phénomène de Stokes en dimension 2", Astérisque 263 (2000), p. 190.

[18] R. G. SWAN - "The theory of sheaves", 1964.

[19] J.-L. VERDIER - "Classe d'homologie associée à un cycle", in Séminaire de géométrie analytique (École Norm. Sup., Paris, 1974-75) Astérisque 36-37 (1976), p. 101-151.

[20] , "Stratifications de Whitney et théorème de Bertini-Sard", Invent. Math. 36 (1976), p. 295-312. 